\title{
Linear Filtering for a Class of Jump Processes Arising in Navigation Systems
}

\author{
T. E. Dabbous \\ Department of Electrical and Electronics Engineering, Bilkent University, Ankara, \\ Turkey \\ S. S. Lim AND N. U. Ahmed \\ Department of Electrical Engineering, University of Ottawa, Ottawa, Canada
}

[Received 19 June 1989 and in revised form 2 March 1990]

\begin{abstract}
In this paper, the filtering problem for a class of jump processes with discrete observations is considered. Using a minimum variance approach, a linear recursive unbiased filter is obtained with the help of which the required estimate and the corresponding covariance can be determined. The proposed filter allows multiple jumps for the state process, thereby making the theory applicable to modern navigation problems (Omega and Loran-C receivers), where multiple jumps have been reported to be a common occurrence. Further, utilizing the filter equations, the question of continuous dependence of the filter on system parameters is studied. Finally, a numerical example based on a navigation system model is presented, to illustrate some of the results of this paper.
\end{abstract}

\section{Introduction}

IN THIS paper, we consider the filtering problem for a class of systems governed by a linear stochastic differential equation of the form

$$
\mathrm{d} x(t)=\tilde{A}(t) \mathrm{d} t+\tilde{\sigma}(t) \mathrm{d} W(t)+\tilde{C} \mathrm{~d} \eta(t) \quad(t \in J=[0, T]), \quad x(0)=x_{0},
$$

where $\bar{A}, \bar{\sigma}$, and $\bar{C}$ are given matrices of appropriate dimensions, $x_{0}$ is a Gaussian random variable, and $W$ is an $n$-dimensional Wiener process independent of $x_{0}$. The process $\{\eta(t): t \geqslant 0\}$, which is independent of $x_{0}$ and $W$, is a temporally homogeneous Markov chain taking values from some finite set. The observation process is given by

$$
y\left(t_{k}\right)=H\left(t_{k}\right) x\left(t_{k}\right)+\left[V\left(t_{k}\right)-V\left(t_{k-1}\right)\right] \quad(k=0,1,2, \ldots, N),
$$

where $V$ is another Wiener process independent of $x_{0}, W$, and $\eta$, and $\left\{t_{k}: 1 \leqslant k \leqslant N\right\}$ is a finite set in $J=[0, T]$ such that $0=t_{0}<t_{1}<\cdots<t_{N}=T$. At each discrete time point $t_{k}$, we have the observation $y\left(t_{k}\right)$ as given above. Our problem is to give an unbiased minimum variance estimate of the process $\{x(t): t \geqslant 0\}$, given the history $\left\{y\left(t_{k}\right): 1 \leqslant k \leqslant N\right\}$.

This problem has been treated in the literature for a special class of jump processes where $\eta$ is only allowed to have single jump $[3,9]$. Here we consider more general situations admitting multiple jumps for the process $\eta$, thereby making the theory applicable to modern navigation problems (Omega and 
Loran- $C$ receivers) where multiple jumps have been reported to be a common occurrence [10].

It is clear from equation (1) that, owing to the presence of the jump process $\eta$, the classical Kalman filter cannot be applied to this problem. In this paper, we develop filter equations that work and give estimates far superior to those obtained by use of a Kalman filter that ignores the presence of jumps. However, as we shall see, if the jump sizes are small, one can ignore the jump process $\eta$ and use a Kalman filter with confidence. On the other hand, if the jump sizes are large, one has to modify the Kalman filter equations so that the effect of these jumps is included in the estimator and covariance (matrix) equations. This fact is clearly indicated in Section 3.

The paper is organized as follows. In Section 2, we formulate the filtering problem and present the necessary assumptions and notation. In Section 3, we use the results of [14] to derive the corresponding filter equations. In Section 4, we utilize the results of Section 3 to study the question of continuous dependence of the filter on system parameters. Finally, in Section 5, we develop an algorithm for computing the required estimate and present a numerical example, which is based on the navigation model developed in [10], to illustrate the effectiveness of the proposed filter.

\section{Formulation of filtering problem, notation, and assumptions}

In navigation problems $[3,9,10]$, the process to be estimated is governed by a system of stochastic differential equations of the form

$$
\mathrm{d} x^{c}(t)=A(t) x^{c}(t) \mathrm{d} t+\sigma(t) \mathrm{d} W(t) \quad(t \in J=[0, T]), \quad x^{c}(0)=x_{0}^{c},
$$

and

$$
\mathrm{d} x^{d}(t)=C(t) \mathrm{d} \eta(t) \quad(t \in J), \quad x^{d}(0)=x_{0}^{d},
$$

where $A \in \mathbb{R}^{n \times n}, \sigma \in \mathbb{R}^{n \times l}$, and $C \in \mathbb{R}^{m \times r}$. The initial states $x_{0}^{c}$ and $x_{0}^{d}$ are assumed to be Gaussian random variables (independent of $W$ and $\eta$ ) with certain mean and covariance. The process $\{W(t): t \in J\}$ is an $l$-dimensional Wiener process satisfying

$$
E\left\{[W(t)-W(s)][W(t)-W(s)]^{\top}\right\}=\int_{s}^{t} Q(\theta) \mathrm{d} \theta \quad(s \leqslant t),
$$

where $Q$ is a positive definite matrix valued function. The process $\{\eta(t): t \in J\}$, which is independent of $x_{0}$ and $W$, is a temporally homogeneous Markov chain taking values from a finite set $\Sigma\left[e_{1}, e_{2}, \ldots, e_{M}\right\} \subset \mathbb{R}^{r}$ with transition probability matrix $S(t)=\left\{S_{t j}(t): i, j=1,2, \ldots, M\right\}(t \geqslant 0)$ satisfying the following (matrix) differential equation

$$
\frac{\mathrm{d}}{\mathrm{d} t} S(t)=\Lambda S(t) \quad(t \in J), \quad S(0)=I,
$$

where $I$ denotes the identity matrix. The matrix $\Lambda$ denotes the infinitesimal 
generator of the Markov chain with elements $\left\{\lambda_{t, j}: i, j=1,2, \ldots, M\right\}$, given by

$$
\lambda_{i, j}= \begin{cases}\lim _{t \downarrow 0} S_{i j}(t) / t & \text { for } i \neq j, \\ \lim _{t \downarrow 0}\left(S_{i j}(t)-1\right) / t & \text { for } i=j .\end{cases}
$$

We assume that the elements $\left\{\lambda_{i, j}\right\}$ satisfy the following property:

$$
\lambda_{i, j}= \begin{cases}\geqslant 0 & \text { for } j=i-1, i+1, \\ -\sum_{k \neq i}^{M} \lambda_{i, k} & \text { for } j=i \\ 0 & \text { otherwise. }\end{cases}
$$

As indicated earlier, a similar class of systems was considered in the literature for filtering navigation signals [3, 9]. For this system, the proces $\eta$ was assumed to satisfy a stochastic differential equation of the form

$$
\mathrm{d} \eta(t)=1\left(\eta\left(t^{-}\right)=0\right) N_{1}(\mathrm{~d} t)-1\left(\eta\left(t^{-}\right)=1\right) N_{2}(\mathrm{~d} t),
$$

where $1(X)$ denotes the indicator function of the set $X$ and $N_{1}$ and $N_{2}$ are two independent Poisson processes with certain mean. Assuming a discrete observation model, the authors in $[3,9]$ have used a minimum variance approach to derive a linear unbiased filter on the basis of which ship (or aircraft) position and velocity can be estimated. In fact, the model given by (6), which has been used in $[3,9]$ to represent the cycle selection error for a Loran-C receiver, was originally proposed by Ahmed \& Dabbous [4] to represent the tie-line behaviour in an interconnected power systems reliability model. Clearly, this model only covers the case where the process $\eta$ has two states only $(0$ or 1$)$. Here we consider the filtering problem for the case where the process $\eta$ is allowed to have multiple jumps. It is clear from equation (5) that, if, for some $t \in J, \eta(t)=e_{i}(1 \leqslant i \leqslant M)$, then, for sufficiently small $\Delta t>0, \eta(t+\Delta t)=e_{i-1}$ or $e_{i}$ or $e_{i+1}$. This fact is indicated in the state transition diagram (Fig. 1).

Using the differential equations (2) and (3), we can write the state (or signal) model as

$$
\mathrm{d} x(t)=\tilde{A}(t) x(t) \mathrm{d} t+\tilde{\sigma}(t) \mathrm{d} W(t)+\tilde{C}(t) \mathrm{d} \eta(t) \quad(t \in J), \quad x(0)=x_{0},
$$

where $x=\left[x^{c}, x^{d}\right]^{\top}$ is a vector in $\mathbb{R}^{n+m}$ and $x_{0}$ is a Gaussian random variable with mean $\bar{x}_{0}$ and covariance $P_{0}$. The matrices $\bar{A} \in \mathbb{R}^{(n+m) \times(n+m)}, \tilde{\sigma} \in \mathbb{P}^{(n+m) \times l}$, and

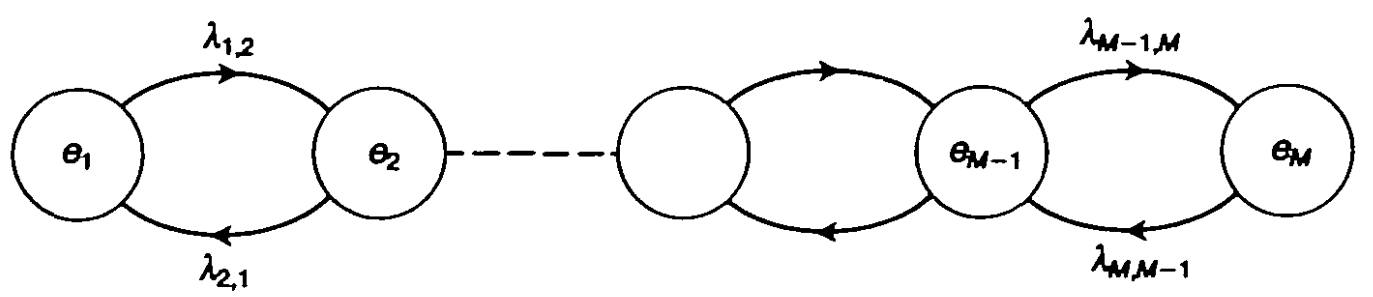

Fio. 1. State transition diagram for $\eta$. 
$\bar{C} \in \mathbb{R}^{(n+m) \times r}$ are, respectively, given by

$$
\bar{A}=\left[\begin{array}{cc}
A & 0 \\
0 & 0
\end{array}\right], \quad \bar{\sigma}=\left[\begin{array}{cc}
\sigma & 0 \\
0 & 0
\end{array}\right], \quad \tilde{C}=\left[\begin{array}{ll}
0 & 0 \\
0 & C
\end{array}\right] .
$$

Thus, the navigation problem is a special case of the general problem as stated in the introduction. Let the observed process be given by

$$
y\left(t_{k}\right)=H\left(t_{k}\right) x\left(t_{k}\right)+\left[V\left(t_{k}\right)-V\left(t_{k-1}\right)\right],
$$

where $V$ is a $2 m$-dimensional Wiener process, independent of $W$ and $\eta$, satisfying

$$
E\left\{\left[V\left(t_{k}\right)-V\left(t_{k-1}\right)\right]\left[V\left(t_{k}\right)-V\left(t_{k-1}\right)\right]^{\top}\right\}=\left(t_{k}-t_{k-1}\right) R\left(t_{k}\right)=\tilde{R}\left(t_{k}\right) .
$$

The matrix $H \in \mathbb{R}^{2 m \times(m+n)}$ is given by

$$
H=\left[\begin{array}{ll}
H_{11} & H_{12} \\
H_{21} & H_{22}
\end{array}\right]
$$

where $H_{11} \in \mathbb{R}^{m \times n}, H_{12} \in \mathbb{R}^{m \times m}, H_{21} \in \mathbb{R}^{m \times n}, H_{22} \in \mathbf{R}^{m \times m}$.

Problem Statement Suppose that the state and observed processes are given by (1) and (8), respectively, and that the processes $W, \eta$, and $V$ are independent. Let $F_{t_{k}}^{y}=\sigma\{y(t): 0 \leqslant i \leqslant k\}$ and suppose that all the above random variables and processes are defined on some complete probability space $(\Omega, F, \mu)$. Then our problem is to determine the conditional expectation of $x$ relative to the output process $y$. That is,

$$
\mathcal{X}\left(t_{k} \mid t_{k}\right)=\mathrm{E}\left\{x\left(t_{k}\right) \mid F_{t_{k}}^{y}\right\} \quad \text { for all } k \text {. }
$$

In the sequel, we shall need the following notation and assumptions.

Notation Let $L_{1}^{\text {loc }}$ denote the class of locally Lebesgue integrable functions on $\mathbb{R}$ such that $\int_{I}|f(t)| \mathrm{d} t<\infty$, for any bounded interval $I \subset R$. Let $F_{t_{k}}^{y}$ denote the $\sigma$-algebra generated by the process $\left\{y\left(t_{i}\right): 0 \leqslant i \leqslant k\right\}$. We use $\sigma\{\eta\}$ to denote the $\sigma$-algebra generated by the random variable $\eta$. Further notation will be introduced in the sequel as required.

\section{Assumptions}

(A1) There exists a positive function $K \in L_{1}^{\text {loc }}$ such that

$$
\|\tilde{A}(t)\| \leqslant K(t) \text { for almost all } t \geqslant 0 \text {. }
$$

(A2) The matrix valued functions $\bar{\sigma}, \bar{C}$, and $Q$ are continuous in $t$ and there exists an $\alpha>0$ such that

$$
(\Gamma \xi, \xi) \geqslant \alpha|\xi|^{2} \text { for all } \xi \in \mathbb{R}^{n+m},
$$

with $\Gamma$ being either of the functions $\tilde{\sigma} \tilde{\sigma}^{\top}$ or $Q$.

It is known that, under the given assumptions, the system (1) has a unique solution with discontinuity of the first kind [12]. In the next section, we shall make use of the results of [14] to obtain a set of differential and difference 
equations that describe the behaviour of the estimator and the corresponding covariance. Utilizing these equations, one can determine (recursively) the required estimate $\hat{x}\left(t_{k} \mid t_{k}\right)$ for all $k$.

\section{Derivation of filter equations}

Consider the systems (1) and (8) and suppose the elements of the matrices $Q$ and $R$ are very small compared to the jump size of the process $x$. Let $\left\{t_{k}: 0 \leqslant k \leqslant N\right\}$ be a finite set in $J \pm[0, T]$ such that $0=t_{0}<t_{1}<\cdots<t_{N}=T$, where $t_{k}$ is the discrete time point at which the observation $y\left(t_{k}\right)$ is available (see equation (8)). Further, during any interval of time $\left[t_{k}, t_{k+1}\right)(1 \leqslant k \leqslant N)$, the process $\eta$ can only make one transition from one state to another and that the infinitesimal rates $\left\{\lambda_{i, j}: i, j=1,2, \ldots, M\right\}$ satisfy the property (5). Under these assumptions, one can determine, by observing $y\left(t_{k}\right)$ and $y\left(t_{k+1}\right)$, whether or not a jump has occurred in $x$ during the time interval $\left(t_{k}, t_{k+1}\right]$. Hence the process $y$ carries information about the jump process $\eta$, and therefore $x$ is conditionally Gaussian relative to $y$. Using this fact, the filtering problem reduces to solving a finite set of differential or difference equations instead of solving linear or nonlinear partial differential equations, as indicated in [5-8, 13].

Since the output process $\left\{y\left(t_{i}\right): i=0,1, \ldots\right\}$ is only available at the discrete points $t_{i}(i=0,1, \ldots)$, the filter will consist of two phases. The first phase is known as the prediction phase, where our estimate is based on predicting the value of $x$ at time $t \in\left[t_{k-1}, t_{k}\right)$ given the output history up to $t_{k-1}$. The second phase is termed the correction phase, in which we correct our prediction as soon as the new observation at time $t_{k}$ becomes available. By this recursive method, one can easily obtain the estimate $\hat{x}\left(t_{k} \mid t_{k}\right)=E\left\{x\left(t_{k}\right) \mid F_{t_{k}}^{y}\right\}$ for all $t_{k}$. In this section, we shall employ the nonlinear filtering formula obtained in [14] to derive the filter equations corresponding to systems (1) and (8). In the following lemma, we present, for convenience, one of the main results of [14].

LEMMA 3.1 [14: Theor. 1] Let $L$ be the (infinitesimal) generator for the state process $x$ and suppose that the observed process is given by

$$
y\left(t_{k}\right)=y_{k}=h_{k}+v_{k} \quad(k=1,2, \ldots, N),
$$

where $h_{k}$ is a certain functional of the state $x(s)\left(s \leqslant t_{k}\right)$ and the observation $y_{l}$, $(l \leqslant k-1)$. Further, $\left\{v_{k}: k \leqslant N\right\}$ is a zero-mean Gaussian white-noise sequence satisfying $\mathrm{E}\left\{v_{k} v_{j}^{\top}\right\}=\delta_{j k} G\left(t_{k}\right)$. Let $g \in D(L)$, where $D(L)$ denotes the domain of $L$, and define

$$
\begin{aligned}
\hat{g}\left(t \mid t_{k-1}\right) & =E\left\{g(t) \mid F_{t_{k-1}}^{y}\right\} & & \left(t \in\left(t_{k-1}, t_{k}\right)\right), \\
\hat{g}\left(t_{k} \mid t\right) & =E\left\{g\left(t_{k}\right) \mid F_{t}^{y}\right\} & & \left(t \in\left(t_{k-1}, t_{k}\right]\right) .
\end{aligned}
$$

Then the estimate $\hat{g}\left(t_{k} \mid t_{k}\right)(1 \leqslant k \leqslant N)$ is determined by the simultaneous solution of the following equations.

(a) Between Observations $\left(t \in\left(t_{k-1}, t_{k}\right)\right)$

$$
\hat{g}\left(t \mid t_{k-1}\right)=\hat{g}\left(t_{k-1} \mid t_{k-1}\right)+\int_{t_{k-1}}^{t} E\left\{(L)(s) \mid F_{t_{k-1}}^{y}\right\} \mathrm{d} s \quad\left(t \in\left(t_{k-1}, t_{k}\right)\right) .
$$


(b) At Observations $\left(t=t_{k}\right)$

$$
\left.\begin{array}{lr}
\left.\frac{\mathrm{d}}{\mathrm{d} t} \boldsymbol{g}\left(t_{k} \mid t\right)=\left[\widehat{\left(g u^{\top}\right.}\right)\left(t_{k} \mid t\right)-\hat{g}\left(t_{k} \mid t\right) \hat{a}^{\top}\left(t_{k} \mid t\right)\right] G^{-1}\left(t_{k}\right)\left[y\left(t_{k}\right)-\hat{h}\left(t_{k} \mid t\right)\right] \\
\hat{g}\left(t_{k} \mid t_{k-1}\right)=\hat{g}\left(t_{k}^{-}\right), & \left(t \in\left(t_{k-1}, t_{k}\right]\right),
\end{array}\right\}
$$

where $u=h_{k} /\left(t_{k}-t_{k-1}\right)$ (see [14: Defn 2 and Remark 1]) and $\hat{g}\left(t_{k}^{-}\right)$is the solution of (9).

Remark 3.1 Using the fact that $F_{s}^{y}=F_{t_{k-1}}^{y}$ for $t_{k-1} \leqslant s<t_{k}$, it follows from (9) that

$$
\frac{\mathrm{d}}{\mathrm{d} t} g(t \mid t)=(\widehat{L g})(t \mid t) \quad\left(t \in\left(t_{k-1}, t_{k}\right)\right),
$$

where $\widehat{(\not g)}(t \mid t)=E\left\{(L g)(t) \mid F_{t}^{y}\right\}$.

With the above result, we shall obtain the filter equations for the systems (1) and (8). For this, we need the following result.

LEMMA 3.2 Consider the system (1) and let $g$ be a twice continuously differentiable function of $x$. Then

$$
\begin{aligned}
& (L g)\left(t, x, e_{i}\right)=(L g)(t) m \lim _{\Delta t \downarrow 0} \frac{1}{\Delta t} \mathrm{E}\left\{g(x(t+\Delta t))-g(x(t)) \mid x(t)=x, \eta(t)=e_{i}\right\} \\
& =\left(g_{x}, \tilde{A}(t) x+\mathcal{C}(t) \sum_{j=1}^{M}\left(e_{j}-e_{i}\right) \lambda_{t, j} 1\left(\eta(t)=e_{i}\right)\right) \\
& \quad+\frac{1}{2} \operatorname{tr}\left(\tilde{\sigma}(t) Q(t) \tilde{\sigma}^{\top}(t)+\sum_{j=1}^{M} \tilde{C}(t)\left(e_{j}-e_{i}\right)\left(e_{j}-e_{i}\right)^{\top} \tilde{C}^{\top}(t) g_{x x} \lambda_{t, j} 1\left(\eta(t)=e_{i}\right)\right),
\end{aligned}
$$

where $(\bullet, \bullet)$ denotes the scalar product, $g_{x}$ is the (partial) derivative of $g$ with respect to $x, \operatorname{tr}(A)$ is the trace of the matrix $A$, and $1(X)$ is the indicator function of the set $X$.

Proof. The proof follows from standard computations and use of Taylor's series expansion.

With the above two lemmas, we have the following theorem.

THEOREM 3.1 (Linear filter for jump processes) Suppose the processes $x$ and $y$ satisfy equations (1) and (8), respectively, and that the assumptions stated above hold. Then the estimate $f\left(t_{k} \mid t_{k}\right)(1 \leqslant k \leqslant N)$ can be determined by the simultaneous solution of the following set of equations.

(a) Prediction Phase

$$
\begin{aligned}
& \frac{\mathrm{d}}{\mathrm{d} t} \hat{x}(t \mid t)=\bar{A}(t) \hat{x}(t \mid t)+\bar{C}(t) \beta^{y}(t), \\
& \frac{\mathrm{d}}{\mathrm{d} t} P(t \mid t)=\bar{A}(t) P(t \mid t)+P(t \mid t) \bar{A}^{\top}(t)+\bar{\sigma}(t) Q(t) \bar{\sigma}^{\top}(t)+\bar{C}(t) \Gamma(t) \bar{C}^{\top}(t),
\end{aligned}
$$


for all $t \in\left[t_{k-1}, t_{k}\right)$, where the initial conditions $P\left(t_{k-1} \mid t_{k-1}\right)$ and $P\left(t_{k-1} \mid t_{k-1}\right)$ are given by (18) and (19) and $\beta^{y}$ and $\Gamma$ are given by

$$
\begin{aligned}
\beta^{y}(t)= & \sum_{i=2}^{M-1}\left[\left(e_{i-1}-e_{i}\right) \lambda_{i, i-1}+\left(e_{i+1}-e_{i}\right) \lambda_{i, i+1}\right] p_{i}^{y}(t) \\
& +\left(e_{2}-e_{1}\right) \lambda_{1,2} p_{1}^{y}(t)+\left(e_{M-1}-e_{M}\right) \lambda_{M, M-1} p_{M}^{y}(t), \\
\Gamma(t)= & \sum_{i=2}^{M-1}\left[\left(e_{i-1}-e_{i}\right)\left(e_{i-1}-e_{i}\right)^{\top} \lambda_{i, i-1}+\left(e_{i+1}-e_{i}\right)\left(e_{i+1}-e_{i}\right)^{\top} \lambda_{i, i+1}\right] \theta_{i}(t) \\
& +\left(e_{2}-e_{1}\right)\left(e_{2}-e_{1}\right)^{\top} \lambda_{1,2} \Theta_{1}(t)+\left(e_{M-1}-e_{M}\right) \\
& \times\left(e_{M-1}-e_{M}\right)^{\top} \lambda_{M, M-1} \Theta_{M}(t),
\end{aligned}
$$

with

$$
\begin{gathered}
p_{i}^{y}(t)=\operatorname{Pr}\left\{\eta(t)=e_{i} \mid F_{t}^{y}\right\} \\
\theta(t)=S(t) \theta_{0}=(\exp \Lambda t) \theta_{0}
\end{gathered}
$$

for all $t \in\left[t_{k-1}, t_{k}\right)$.

(b) Correction Phase

$$
\begin{aligned}
f\left(t_{k} \mid t_{k}\right) & =\mathcal{f}\left(t_{k} \mid t_{k-1}\right)+G^{0}\left(t_{k}\right)\left[y\left(t_{k}\right)-H\left(t_{k}\right) \mathcal{P}\left(t_{k} \mid t_{k-1}\right)\right], \\
P\left(t_{k} \mid t_{k}\right) & =\left[I-G^{0}\left(t_{k}\right) H\left(t_{k}\right)\right] P\left(t_{k} \mid t_{k-1}\right), \\
G^{0}\left(t_{k}\right) & =P\left(t_{k} \mid t_{k-1}\right) H^{\top}\left(t_{k}\right)\left[H\left(t_{k}\right) P\left(t_{k} \mid t_{k-1}\right) H^{\top}\left(t_{k}\right)+\tilde{R}\left(t_{k}\right)\right]^{-1},
\end{aligned}
$$

where $\hat{L}\left(t_{k} \mid t_{k-1}\right)$ and $P\left(t_{k} \mid t_{k-1}\right)$ are the solutions of (12) and (13), respectively.

Proof. For the prediction phase, we shall show that equations (12) and (13) follow from $\left(10^{\prime}\right)$ and (11). Setting $g(x)=x_{\alpha}(1 \leqslant \alpha \leqslant m+n)$, it follows from $\left(10^{\prime}\right)$ and (11) that

$$
\frac{\mathrm{d}}{\mathrm{d} t} \hat{x}_{\alpha}(t \mid t)=\sum_{q=1}^{m+n} \bar{A}_{\alpha q q}(t) \hat{x}_{q}(t \mid t)+\sum_{q=1}^{r} \sum_{i, j=1}^{M} \bar{C}_{\alpha q}(t)\left(e^{q}-e^{q}\right) \lambda_{i, j} p_{i}^{y}(t)
$$

for all $t \in\left(t_{k-1}, t_{k}\right)$ and $1 \leqslant \alpha \leqslant m+n$, where $e q$ denotes the $q$ th component of the vector $e_{j}$ and $\left\{p_{i}^{y}: 1 \leqslant i \leqslant M\right\}$ is given by (16). Using the property (5) of the Markov chain $\eta$, one can easily verify that (12) follows from (21). Next, if we define

$$
P_{\alpha \beta}(t \mid t)=\mathrm{E}\left\{\left[x_{\alpha}(t)-\hat{x}_{\alpha}(t \mid t)\right]\left[x_{\beta}(t)-\hat{x}_{\beta}(t \mid t)\right]\right\} \quad(1 \leqslant \alpha, \beta \leqslant m+n),
$$

then it is clear that

$$
\left.P_{\alpha \beta}(t \mid t)=\mathrm{E}\left\{\widehat{\left(x_{\alpha} x_{\beta}\right.}\right)(t \mid t)-\hat{x}_{\alpha}(t \mid t) \hat{x}_{\beta}(t \mid t)\right\} \quad(1 \leqslant \alpha, \beta \leqslant m+n)
$$

for all $t \in\left(t_{k-1}, t_{k}\right)$. Setting $g(x)=x_{\alpha} x_{\beta}$ and using $\left(10^{\prime}\right)$ and (11), we have

$$
\begin{aligned}
\frac{\mathrm{d}}{\mathrm{d} t}\left(\widehat{x_{\alpha} x_{\beta}}\right)(t \mid t)= & \left.\left.\sum_{q=1}^{m+n}\left[\bar{A}_{\alpha q}(t) \widehat{\left(x_{q} x_{\beta}\right.}\right)(t \mid t)+\bar{A}_{\beta q}(t) \widehat{\left(x_{q} x_{\alpha}\right.}\right)(t \mid t)\right] \\
& +\sum_{q=1}^{r} \sum_{i, j=1}^{M} \bar{C}_{\alpha q}(t)(e q-e q) \hat{x}_{\beta}(t \mid t) \lambda_{i, j} p_{i}^{y}(t)
\end{aligned}
$$




$$
\begin{aligned}
& +\sum_{q=1}^{r} \sum_{i, j=1}^{M} \tilde{C}_{\beta q}(t)(e q-e q) \tilde{x}_{\alpha}(t \mid t) \lambda_{i, j} p_{i}^{y}(t) \\
& +\sum_{q, s=1}^{t} \bar{\sigma}_{\alpha q}(t) Q_{q s}(t) \tilde{\sigma}_{\beta s}(t) \\
& +\sum_{q, s=1}^{r} \sum_{i, j=1}^{M} \bar{C}_{\alpha q g}(t)(e q-e q)\left(e_{j}^{s}-e_{i}^{s}\right) \bar{C}_{\beta v}(t) \lambda_{t, j} \\
& \times E\left\{1\left(\eta(t)=e_{i}\right) \mid F_{t}^{y}\right\}
\end{aligned}
$$

for all $t \in\left(t_{k-1}, t_{k}\right)$. Using (21)-(23) and noting that

$$
\mathrm{E}\left\{\mathrm{E}\left\{1\left(\eta(t)=e_{i}\right) \mid F_{i}^{y}\right\}\right\}=\mathrm{E}\left\{1\left(\eta(t)=e_{i}\right)\right\}=\operatorname{Pr}\left\{\eta(t)=e_{i}\right\}=\theta_{i}(t),
$$

one can easily verify that

$$
\begin{aligned}
\frac{\mathrm{d}}{\mathrm{d} t} P_{\alpha \beta}(t \mid t)= & \sum_{q=1}^{m+n}\left[\bar{A}_{\alpha q}(t) P_{q \beta}(t \mid t)+\bar{A}_{\beta q}(t) P_{q \alpha}(t \mid t)\right] \\
& +\sum_{q, s=1}^{t} \bar{\sigma}_{\alpha q q}(t) Q_{q s}(t) \bar{\sigma}_{\beta s}(t) \\
& +\sum_{q, s=1}^{r} \bar{C}_{\alpha r t}(t)(e q-e q)\left(e_{j}^{s}-e_{t}^{s}\right) \tilde{C}_{\beta s}(t) \lambda_{t, j} \Theta_{i}(t)
\end{aligned}
$$

for $t \in\left(t_{k-1}, t_{k}\right)$. Now, using (5) and (24), one obtains (13). To complete the proof of the theorem, we need to show that (18) and (19) follow from (10). Indeed, setting $h_{k} \equiv H\left(t_{k}\right) x\left(t_{k}\right)$, replacing $G^{-1}\left(t_{k}\right)$ by $\bar{R}^{-1}\left(t_{k}\right)=$ $R^{-1}\left(t_{k}\right) /\left(t_{k}-t_{k-1}\right)$, and setting $g(x)=x_{\alpha}(1 \leqslant \alpha \leqslant m+n)$, we find from (10) that

$$
\left.\begin{array}{lr}
\left.\frac{\mathrm{d}}{\mathrm{d} t} \hat{x}_{\alpha}\left(t_{k} \mid t\right)=\left[\widehat{\left(x_{\alpha} x^{\top}\right.}\right)\left(t_{k} \mid t\right)-\hat{x}_{\alpha}\left(t_{k} \mid t\right) \hat{R}^{\top}\left(t_{k} \mid t\right)\right] H^{\top}\left(t_{k}\right) \tilde{R}^{-1}\left(t_{k}\right) D\left(t_{k}\right) \\
\hat{x}_{\alpha}\left(t_{k} \mid t_{k-1}\right)=\hat{x}_{\alpha}\left(t_{k}^{-}\right), & \left(t \in\left(t_{k-1}, t_{k}\right]\right),
\end{array}\right\}
$$

where $\hat{V}\left(t_{k}\right)=y\left(t_{k}\right)-H\left(t_{k}\right) \hat{x}\left(t_{k} \mid t\right)$ denotes the innovation process. We define

$$
\begin{aligned}
P_{\alpha \beta}\left(t_{k} \mid t\right) & =\mathrm{E}\left\{\left[x_{\alpha}\left(t_{k}\right)-\hat{x}_{\alpha}\left(t_{k} \mid t\right)\right]\left[x_{\beta}\left(t_{k}\right)-\hat{x}_{\beta}\left(t_{k} \mid t\right)\right] \mid F_{t}^{y}\right\} \\
& =\left(\widehat{x_{\alpha} x_{\beta}}\right)\left(t_{k} \mid t\right)-\hat{x}_{\alpha}\left(t_{k} \mid t\right) \hat{x}_{\beta}\left(t_{k} \mid t\right)
\end{aligned}
$$

for all $t \in\left(t_{k-1}, t_{k}\right]$ and $1 \leqslant \alpha, \beta \leqslant m+n$. We shall show later that the conditional covariance $P\left(t_{k} \mid t\right)$, whose components are given by (26), is independent of the observed process $y$ and hence the conditional and unconditional covariance matrices are equal. Setting $g(x)=x_{\alpha} x_{\beta}$ in (10), it follows that

$$
\frac{\mathrm{d}}{\mathrm{d} t}\left(\widehat{x_{\alpha} x_{\beta}}\right)\left(t_{k} \mid t\right)=\left[\left(\widehat{\left(x_{\alpha} x_{\beta} x^{\top}\right.}\right)\left(t_{k} \mid t\right)-\left(\widehat{x_{\alpha} x_{\beta}}\right)\left(t_{k} \mid t\right) \hat{R}^{\top}\left(t_{k} \mid t\right)\right] H^{\top}\left(t_{k}\right) \tilde{R}^{-1}\left(t_{k}\right) \nabla\left(t_{k}\right)
$$

for all $t \in\left(t_{k-1}, t_{k}\right]$. Now using (25), (26), and (26'), and noting that, under the 
hypotheses of the theorem, the process $P\left(t_{k}\right)$ is Gaussian, one can verify that

$$
\begin{aligned}
& \frac{\mathrm{d}}{\mathrm{d} t} P_{\alpha \beta}\left(t_{k} \mid t\right)=\hat{X}\left(t_{k} \mid t\right) H^{\top}\left(t_{k}\right) \widetilde{R}^{-1}\left(t_{k}\right) D\left(t_{k}\right) \\
& \left.\quad-\left[\widehat{\left(x_{\alpha} x\right)}\left(t_{k} \mid t\right)-\hat{x}_{\alpha}\left(t_{k} \mid t\right) \hat{R}\left(t_{k} \mid t\right)\right]^{\top} \tilde{H}\left(t_{k}\right)\left[\widehat{\left(x_{\beta} x\right.}\right)\left(t_{k} \mid t\right)-\hat{x}_{\beta}\left(t_{k} \mid t\right) \hat{X}\left(t_{k} \mid t\right)\right],
\end{aligned}
$$

where $\bar{H} \equiv H^{\top} \bar{R}^{-1} H$ and

$$
\mathcal{X}\left(t_{k} \mid t\right)=\mathrm{E}\left\{\left[x_{\alpha}\left(t_{k}\right)-\hat{x}_{\alpha}\left(t_{k} \mid t\right)\right]\left[x_{\beta}\left(t_{k}\right)-\hat{x}_{\beta}\left(t_{k} \mid t\right)\right]\left[x\left(t_{k}\right)-\hat{x}\left(t_{k} \mid t\right)\right]^{\top} \mid F_{t}^{y}\right\} .
$$

Since, as indicated earlier, the process $x$ is conditionally Gaussian relative to $y$, it follows that

$$
\begin{aligned}
& \frac{\mathrm{d}}{\mathrm{d} t} P_{\alpha \beta}\left(t_{k} \mid t\right) \\
& \quad=-\left[\widehat{\left(x_{\alpha} x\right)}\left(t_{k} \mid t\right)-\hat{x}_{\alpha}\left(t_{k} \mid t\right) \hat{x}\left(t_{k} \mid t\right)\right]^{\top} \tilde{H}\left(t_{k}\right)\left[\widehat{\left(x_{\beta} x\right)}\left(t_{k} \mid t\right)-\hat{x}_{\beta}\left(t_{k} \mid t\right) \hat{x}\left(t_{k} \mid t\right)\right]
\end{aligned}
$$

for all $1 \leqslant \alpha, \beta \leqslant m+n$. Hence, it is clear that

$$
\frac{\mathrm{d}}{\mathrm{d} t} P\left(t_{k} \mid t\right)=-P\left(t_{k} \mid t\right) \tilde{H}\left(t_{k}\right) p\left(t_{k} \mid t\right) \quad\left(t \in\left(t_{k-1}, t_{k}\right]\right), \quad P\left(t_{k-1} \mid t_{k}\right)=P\left(t_{k}^{-}\right),
$$

where $P\left(t_{k} \mid t\right)=E\left\{\left[x\left(t_{k}\right)-\hat{x}\left(t_{k} \mid t\right)\right]\left[x\left(t_{k}\right)-\hat{x}\left(t_{k} \mid t\right)\right]^{\top} \mid F_{t}^{y}\right\}$ for all $t \in\left(t_{k-1}, t_{k}\right]$. From (28), it is clear that $P\left(t_{k} \mid t\right)$ is independent of $y$, and hence

$$
\begin{aligned}
P\left(t_{k} \mid t\right) & =\mathrm{E}\left\{\left[x\left(t_{k}\right)-\hat{x}\left(t_{k} \mid t\right)\right]\left[x\left(t_{k}\right)-\hat{x}\left(t_{k} \mid t\right)\right]^{\top} \mid F_{t}^{y}\right\} \\
& =\mathrm{E}\left\{\left[x\left(t_{k}\right)-\hat{x}\left(t_{k} \mid t\right)\right]\left[x\left(t_{k}\right)-\hat{x}\left(t_{k} \mid t\right)\right]^{\top}\right\} \quad\left(t \in\left(t_{k-1}, t_{k}\right]\right) .
\end{aligned}
$$

Using the definition of $P\left(t_{k} \mid t\right)$, it follows from (25) that

$$
\frac{\mathrm{d}}{\mathrm{d} t} \boldsymbol{x}\left(t_{k} \mid t\right)=P\left(t_{k} \mid t\right) H^{\top}\left(t_{k}\right) \tilde{R}^{-1}\left(t_{k}\right) \hat{P}\left(t_{k}\right), \quad \hat{x}\left(t_{k-1} \mid t\right)=\hat{x}\left(t_{k}^{-}\right)
$$

for $t \in\left(t_{k-1}, t_{k}\right]$. It is not difficult to see that the solutions of (28) and (29) are, respectively, given by

$$
\begin{aligned}
& P\left(t_{k} \mid t_{k}\right)=P\left(t_{k} \mid t_{k-1}\right)-G^{0}\left(t_{k}\right) H\left(t_{k}\right) P\left(t_{k} \mid t_{k-1}\right), \\
& \hat{x}\left(t_{k} \mid t_{k}\right)=\hat{x}\left(t_{k} \mid t_{k-1}\right)+G^{0}\left(t_{k}\right) \mathcal{P}\left(t_{k}\right),
\end{aligned}
$$

where $G^{0}$ is given by (20). This completes the proof.

Remark 3.2 Note that, in order to use (12) for determining the estimate $\hat{x}(t \mid t)\left(t \in\left(t_{k-1}, t_{k}\right)\right)$, one is required to compute the quantity $\beta^{y}$ (see equation (14)), which requires computing the conditional probabilities $\left\{p_{l}^{y}: 1 \leqslant i \leqslant M\right\}$. An explicit expression for these conditional probabilities is rather hard to obtain. However, with the assumption that the elements of the covariance matrices $Q$ and $R$ are very small compared to the jump size of the process $x$, one can estimate with a high degree of reliability the values of $p_{i}^{y}(1 \leqslant i \leqslant M)$ with the help of the logic given in the algorithm presented in Section 5 (see Step 3). This logic is based on arguments similar to those given in [3]. 
Remark 3.3 Suppose that the process $\eta$ is governed by the (scalar) stochastic differential equation

$$
\mathrm{d} \eta(t)=1\left(\eta\left(t^{-}\right)=0\right) N_{1}(\mathrm{~d} t)-1\left(\eta\left(t^{-}\right)=1\right) N_{2}(\mathrm{~d} t) \quad(t \geqslant 0),
$$

where $N_{1}$ and $N_{2}$ are two independent Poisson processes with means $\lambda_{1,2}$ and $\lambda_{2,1}$, respectively. In this case, the set $\Sigma=\{0,1\}$. Setting $e_{1}=0, e_{2}=1$, and $M=2$, it follows from equations (14) and (15) that

$$
\beta^{y}(t)=\lambda_{1,2} p_{1}^{y}(t)-\lambda_{2,1} p_{2}^{y}(t) \text { and } \Gamma(t)=\lambda_{1,2} \theta_{1}(t)+\lambda_{2,1} \Theta_{2}(t)
$$

The above (scalar) expressions were obtained in [3] for the case where the process $\eta$ has only two states ( 0 or 1$)$.

Remark 3.4 Setting $\bar{C}(t)=0$ for all $t \geqslant 0$ in equations (12) and (13), we obtain the usual Kalman filter for a continuous state with discrete observations (see e.g. [11]).

In the next section, we utilize the result of Theorem 3.1 to study the question of continuous dependence of the filter on the system parameters $\bar{x}_{0}, P_{0}, \tilde{A}, \tilde{\sigma}, \tilde{C}$, $Q$, and the infinitesimal generator $\Lambda$ of the Markov chain.

\section{Continuous dependence of the filter on system parameters}

The question of continuous dependence of solutions on system parameters (robustness) plays a central role in the study of sensitivity, stability, and optimal control [1]. This question arises when the system parameters are subjected to perturbations or because of the neglected dynamics in the mathematical models. In the filtering problem, this question arises because the filter parameters (i.e. $\bar{A}$, $\tilde{C}, \tilde{\sigma}, Q$, and $\Lambda$ ) are usually determined by field measurement. Hence any error in these measurements will cause errors in the estimate $\hat{f}$ as well as the corresponding covariance $P$. This problem will automatically lead us to the question of system identification (or adaptive filtering), which in turn requires the study of the continuity as well as differentiability of the filter with respect to these parameters. In this section, we present some results that show the continuity of the proposed filter (see Theorem 3.1) with respect to the initial data $\bar{x}_{0}$ and $P_{0}$ as well as the parameters $\tilde{A}, \tilde{C}, \bar{\sigma}, Q$, and the infinitesimal generator $\Lambda$ of the Markov chain. For convenience of presentation, we shall prove these results for a fixed but arbitrary interval $J \equiv\left[0, t_{1}\right]$. However, the results obtained are valid for any finite time interval. Further, for notational convenience from now on we shall use $\beta$ and $p$ to denote $\beta^{y}$ and $p^{y}$, respectively (see (14)).

This section is organized as follows. We shall first prove the continuity of the filter with respect to the parameter $\Lambda$. Then, using similar arguments, we prove the continuity with respect to $\bar{x}_{0}, P_{0}, \tilde{A}, \bar{\sigma}$, and $Q$. In all the proofs, we shall follow similar arguments as those given in [1] or [2].

Let $P$ denote the class of parameters $\left\{\lambda_{t, 1}\right\}$ satisfying the property (5). Let $\lambda^{k}, \lambda^{0} \in P$ and let $\hat{x}^{k}(t)=\hat{x}^{k}(t \mid 0), \quad \hat{x}^{0}(t)=\hat{x}^{0}(t \mid 0), \quad P^{k}(t)=P_{k}(t \mid 0), \quad$ and $P^{0}(t)=P^{0}(t \mid 0)$, denote the corresponding solutions of the differential equations (12) and (13) for all $t \in J=\left[0, t_{1}\right]$. The following result shows that $x^{k}(t) \rightarrow \hat{x}^{0}(t)$ and $P^{k}(t) \rightarrow P^{0}(t)$ uniformly in $t \in J$ whenever $\lambda^{k} \rightarrow \lambda^{0}$ in $P$. 
THEOREM 4.1 (Continuous dependence on $\Lambda$ ) Consider the filter equations (12) and (13) and suppose Assumptions (A1) and (A2) hold. Let $\lambda^{k}\left\{\lambda_{i, j}^{k}: i, j=\right.$ $1,2, \ldots, M\}$ be a sequence in $P$ such that $\lambda^{k} \rightarrow \lambda^{0} \in P$. Then $x^{k}(t) \rightarrow \ell^{0}(t)$ and $P^{k}(t) \rightarrow P^{0}(t)$ uniformly in $t \in J$.

The proof of the above Theorem is based on the following Lemmas.

LEMMA 4.1 Let $\lambda^{k}, \lambda^{0} \in P$ and let $\beta^{k}$ and $\beta^{0}$ be given by (14), with $\lambda$ being replaced by $\lambda^{k}$ and $\lambda^{0}$, respectively. Then $\beta^{k}(t) \rightarrow \beta^{0}(t)$ uniformly in $t \in J$ whenever $\lambda^{k} \rightarrow \lambda^{0} \in P$.

Proof. For $t \in J$, define

$$
\begin{aligned}
\beta^{k}(t)= & \sum_{i=2}^{N-1}\left[\left(e_{i-1}-e_{i}\right) \lambda_{i, i-1}^{k}+\left(e_{i+1}-e_{i}\right) \lambda_{i, i+1}^{k}\right] p_{i}(t) \\
& +\left(e_{2}-e_{1}\right) \lambda_{1,2}^{k} p_{1}(t)+\left(e_{M-1}-e_{M}\right) \lambda_{M, M-1}^{k} p_{M}(t), \\
\beta^{0}(t) \equiv & \sum_{i=2}^{M-1}\left[\left(e_{i-1}-e_{i}\right) \lambda_{i, i-1}^{0}+\left(e_{i+1}-e_{i}\right) \lambda_{i, i+1}^{0}\right] p_{i}(t) \\
& +\left(e_{2}-e_{1}\right) \lambda_{1,2}^{0} p_{1}(t)+\left(e_{M-1}-e_{M}\right) \lambda_{M, M-1}^{0} p_{M}(t) .
\end{aligned}
$$

Then it is clear that

$$
\begin{aligned}
\sup _{t \in}\left|\beta^{k}(t)-\beta^{0}(t)\right| \leqslant \sum_{i=2}^{M-1}( & \left.\left|e_{i-1}-e_{i}\right|\left|\lambda_{i, i-1}^{k}-\lambda_{i, i-1}^{0}\right|+\left|e_{i+1}-e_{i}\right|\left|\lambda_{i, l+1}^{k}-\lambda_{i, i+1}^{0}\right|\right) \\
& +\left|e_{2}-e_{1}\right|\left|\lambda_{1,2}^{k}-\lambda_{1,2}^{0}\right|+\left|e_{M}-e_{M-1}\right|\left|\lambda_{M, M-1}^{k}-\lambda_{M, M-1}^{0}\right|,
\end{aligned}
$$

and hence the result follows.

LEMMA 4.2 Consider the (matrix) differential equation

$$
\frac{\mathrm{d}}{\mathrm{d} t} S(t)=\Lambda S(t) \quad(t \in J), \quad S(0)=I,
$$

where the elements of $\Lambda \in \mathbb{R}^{M \times M}$ are given by $\left\{\lambda_{i, j}: i, j=1,2, \ldots, M\right\}$. Let $P_{\Lambda}$ denote the class of matrices in $\mathbb{R}^{M \times M}$ whose elements satisfy property (5). Then the map $\Lambda \rightarrow S(\cdot, \Lambda)$ is continuous on $P_{\Lambda}$.

Proof. Let $\Lambda^{k}, \Lambda^{0} \in P_{\Lambda}$ such that $\Lambda^{k} \rightarrow \Lambda^{0}$ and let $S^{k}(t) m S\left(t, \Lambda^{k}\right)$ and $S^{0}(t) \equiv S\left(t, \Lambda^{0}\right)(t \in J)$ denote the solutions of (31) corresponding to $\Lambda^{k}$ and $\Lambda^{0}$, respectively. Then

$$
S^{k}(t)=I+\Lambda^{k} \int_{0}^{t} S^{k}(\theta) \mathrm{d} \theta \quad(t \in J)
$$

By Gronwall's lemma, it follows that

$$
\sup _{t \in f}\left\|S^{k}(t)\right\| \leqslant \exp \left(t_{1}\left\|\Lambda^{k}\right\|\right)=b_{1}<\infty .
$$

Since

$$
S^{0}(t)=I+\Lambda^{0} \int_{0}^{t} S^{0}(\theta) \mathrm{d} \theta \quad(t \in J)
$$


using the estimate (32) and Gronwall's lemma, one can easily verify that

$$
\sup _{t \in f}\left\|S^{k}(t)-S^{0}(t)\right\| \leqslant\left[b_{1} \exp \left(t_{1}\left\|\Lambda^{0}\right\|\right)\right]\left\|\Lambda^{k}-\Lambda^{0}\right\|,
$$

and the result follows.

Lemma 4.3 Let $\lambda^{k}, \lambda^{0} \in \mathcal{P}$ be such that $\lambda^{k} \rightarrow \lambda^{0}$. For every $t \in J$, define

$$
\begin{aligned}
\Gamma^{*}(t)= & \sum_{i=2}^{M-1}\left[\left(e_{i-1}-e_{i}\right)\left(e_{t-1}-e_{i}\right)^{\top} \lambda_{i, i-1}^{k}+\left(e_{i+1}-e_{i}\right)\left(e_{i+1}-e_{i}\right)^{\top} \lambda_{i, i+1}^{k}\right] \Theta_{i}^{k}(t) \\
& +\left(e_{2}-e_{1}\right)\left(e_{2}-e_{1}\right)^{\top} \lambda_{1,2}^{k} \theta_{1}^{k}(t)+\left(e_{M-1}-e_{M}\right)\left(e_{M-1}-e_{M}\right)^{\top} \lambda_{M, M-1}^{k} \theta_{M}^{k}(t), \\
\Gamma^{0}(t)= & \sum_{i=2}^{M-1}\left[\left(e_{t-1}-e_{i}\right)\left(e_{i-1}-e_{i}\right)^{\top} \lambda_{i, i-1}^{0}+\left(e_{i+1}-e_{i}\right)\left(e_{i+1}-e_{i}\right)^{\top} \lambda_{i, i+1}^{0}\right] \Theta_{i}^{0}(t) \\
& +\left(e_{2}-e_{1}\right)\left(e_{2}-e_{1}\right)^{\top} \lambda_{1,2}^{0} \theta_{1}^{0}(t)+\left(e_{M-1}-e_{M}\right)\left(e_{M-1}-e_{M}\right)^{\top} \lambda_{M, M-1}^{0} \Theta_{M}^{0}(t) .
\end{aligned}
$$

Then $\Gamma^{\star}(t) \rightarrow \Gamma^{0}(t)$ uniformly in $t \in J$ whenever $\lambda^{k} \rightarrow \lambda^{0}$.

Proof. Since $\Theta^{k}(t)=S^{k}(t) \Theta_{0}$ and $\Theta^{0}(t)=S^{0}(t) \Theta_{0}$ for all $t \in J$, it follows from Lemma 4.2 that $\theta^{k}(t) \rightarrow \Theta^{0}(t)$ uniformly in $t \in J$. Using (33) and (34), one can easily verify that

$$
\begin{aligned}
\sup _{t \in}\left\|\Gamma^{k}(t)-\Gamma^{0}(t)\right\| \leqslant & \sum_{i=2}^{M-1}\left[\alpha_{i-1}\left|\lambda_{i, t-1}^{k}-\lambda_{i, t-1}^{0}\right|\right. \\
& \left.+\left(\tilde{\alpha}_{i-1}+\tilde{\alpha}_{i+1}\right) \sup _{t \in}\left|\Theta_{i}^{k}(t)-\Theta_{i}^{0}(t)\right|+\alpha_{i+1}\left|\lambda_{i, t+1}^{k}-\lambda_{i, t+1}^{0}\right|\right] \\
& +\alpha_{1}\left|\lambda_{1,2}^{k}-\lambda_{1,2}^{0}\right|+\bar{\alpha}_{1} \sup _{t \in}\left|\Theta_{1}^{k}(t)-\Theta_{1}^{0}(t)\right| \\
& +\alpha_{M}\left|\lambda_{M, M-1}^{k}-\lambda_{M, M-1}^{0}\right|+\tilde{\alpha}_{M} \sup _{t \in}\left|\theta_{M}^{k}(t)-\Theta_{M}^{0}(t)\right|,
\end{aligned}
$$

where $\alpha_{i}$ and $\bar{\alpha}_{i}(1 \leqslant i \leqslant M)$ are some positive constants. From the inequality (35), it is clear that $\Gamma^{\star}(t) \rightarrow \Gamma^{0}(t)$ uniformly in $t \in J$ as $k \rightarrow \infty$. This completes the proof.

With the help of the above lemmas, we now prove Theorem 4.1.

Proof of Theorem 4.1. Let $\lambda^{k}, \lambda^{0} \in P$. For $t \in J$, define

$$
\begin{aligned}
& \hat{x}^{k}(t)=\bar{x}_{0}+\int_{0}^{t} \bar{A}(\theta) \hat{x}^{k}(\theta) \mathrm{d} \theta+\int_{0}^{t} \tilde{C}(\theta) \beta^{k}(\theta) \mathrm{d} \theta, \\
& \hat{x}^{0}(t)=\bar{x}_{0}+\int_{0}^{t} \bar{A}(\theta) \hat{x}^{0}(\theta) \mathrm{d} \theta+\int_{0}^{t} \tilde{C}(\theta) \beta^{0}(\theta) \mathrm{d} \theta .
\end{aligned}
$$


Hence

$$
\left|\hat{R}^{k}(t)-\hat{\ell}^{0}(t)\right| \leqslant \int_{0}^{t}\|\tilde{A}(\theta)\|\left|\hat{\mathfrak{R}}^{k}(\theta)-\mathfrak{R}^{0}(\theta)\right| \mathrm{d} \theta+\int_{0}^{t}\|\tilde{C}(\theta)\|\left|\beta^{k}(\theta)-\beta^{0}(\theta)\right| \mathrm{d} \theta
$$

Using Assumptions (A1)-(A2) and Gronwall's lemma, one can easily verify that

$$
\sup _{t \in f}\left|\hat{\beta}^{k}(t)-\hat{\ell}^{0}(t)\right| \leqslant b_{2} \exp \left(\int_{0}^{t_{1}} K(\theta) \mathrm{d} \theta\right) \int_{0}^{t_{1}}\left|\beta^{k}(\theta)-\beta^{0}(\theta)\right| \mathrm{d} \theta
$$

where $b_{2}=\sup _{t \in J}\left\|C^{X}(t)\right\|$. Since $K \in L_{1}^{\text {loc }}$, supreJ $\left|\beta^{k}\right|<\infty, \int_{0}^{t_{1}}\left|\beta^{k}(t)\right| \mathrm{d} t<\infty$, and by Lemma $4.1 \beta^{k}(t) \rightarrow \beta^{0}(t)$ for every $t \in J$, it follows from the dominated convergence theorem that the right-hand side of (38) converges to zero in the limit. Hence we conclude that $\hat{x}^{k}(t) \rightarrow \hat{x}^{0}(t)$ uniformly in $t \in J$.

To complete the proof of the theorem, it remains to show that $P^{k}(t) \rightarrow P^{0}(t)$ uniformly in $t \in J$. Indeed, using (13), we have

$$
\begin{aligned}
& P^{k}(t)=P_{0}+\int_{0}^{t} \tilde{A}(\theta) P^{k}(\theta) \mathrm{d} \theta+\int_{0}^{t} P^{\star}(\theta) \tilde{A}^{\top}(\theta) \mathrm{d} \theta \\
&+\int_{0}^{t} \tilde{\sigma}(\theta) Q(\theta) \tilde{\sigma}^{\top}(\theta) \mathrm{d} \theta+\int_{0}^{t} \tilde{C}(\theta) \Gamma^{\star}(\theta) \bar{C}^{\top}(\theta) \mathrm{d} \theta, \\
& P^{0}(t)=P_{0}+\int_{0}^{t} \tilde{A}(\theta) P^{0}(\theta) \mathrm{d} \theta+\int_{0}^{t} P^{0}(\theta) \tilde{A}^{\top}(\theta) \mathrm{d} \theta \\
&+\int_{0}^{t} \tilde{\sigma}(\theta) Q(\theta) \tilde{\sigma}^{\top}(\theta) \mathrm{d} \theta+\int_{0}^{t} \bar{C}(\theta) \Gamma^{0}(\theta) \bar{C}^{\top}(\theta) \mathrm{d} \theta,
\end{aligned}
$$

for all $t \in J$. Again by Assumptions (A1) and (A2) and Gronwall's lemma, it follows that there exists a number $0<b_{3}<\infty$ such that

$$
\sup _{s e}\left\|P^{k}(t)-P^{0}(t)\right\| \leqslant b_{3} \exp \left(2 \int_{0}^{t_{1}} K(\theta) \mathrm{d} \theta\right) \int_{0}^{t_{1}}\left\|\Gamma^{\star}(\theta)-\Gamma^{0}(\theta)\right\| \mathrm{d} \theta .
$$

Since $K \in L_{1}^{\text {bo }}$, $\sup _{t e J}\left\|\Gamma^{\star}(t)\right\|<\infty, \int_{J}\left\|\Gamma^{\star}(t)\right\| \mathrm{d} t<\infty$, and by Lemma $4.3 \Gamma^{\star}(t) \rightarrow$ $\Gamma^{0}(t)$ for every $t \in J$, it follows from the dominated convergence theorem that the right-hand side of (39) converges to zero as $k \rightarrow \infty$. This ends the proof of Theorem 4.1.

In the remaining part of this section, we shall use arguments similar to those given above to prove the continuity of the mappings $\left(\bar{x}_{0}, \tilde{A}, \tilde{C}\right) \rightarrow \mathfrak{x}\left(\bullet, \bar{x}_{0}, \tilde{A}, \tilde{C}\right)$ and $\left(P_{0}, \bar{A}, \tilde{\sigma}, Q, \tilde{C}\right) \rightarrow P\left(\bullet, P_{0}, \tilde{A}, \tilde{\sigma}, Q, \bar{C}\right)$, where $\hat{x}\left(t, \bar{x}_{0}, \bar{A}, \bar{C}\right)$ and $P\left(t, P_{0}\right.$, $\tilde{A}, \tilde{\sigma}, Q, \tilde{C})(t \in J)$ denote the solutions of equations (12) and (13), respectively, corresponding to the parameters $\bar{x}_{0}, P_{0}, \tilde{A}, \tilde{\sigma}, Q$, and $\bar{C}$.

THEOREM 4.2 Consider the filter equations (12) and (13) and suppose that Assumptions (A1) and (A2) hold. Let $\left(\bar{x}_{0}^{k}, P_{0}^{k}, \bar{A}^{k}, \tilde{\sigma}^{k}, Q^{k}, \bar{C}^{k}\right)$ be a sequence of parameters such that

$$
\begin{array}{ll}
\bar{x}_{0}^{k} \rightarrow \bar{x}_{0}^{0} & \text { in } \mathbb{R}^{n+m}, \\
P_{0}^{k} \rightarrow P_{0}^{0} & \text { in } \mathbb{R}^{(n+m) \times(n+m)}, \\
\tilde{A}^{k}(t) \rightarrow \bar{A}^{0}(t) & \text { for almost all } t \in J, \\
\left\{\tilde{\sigma}^{k}(t), Q^{k}(t), \tilde{C}^{k}(t)\right\} \rightarrow\left\{\tilde{\sigma}^{0}(t), Q^{0}(t), \tilde{C}^{0}(t)\right\} \quad \text { uniformly in } t \in J .
\end{array}
$$


Let $\hat{x}^{k}(t), \hat{x}^{0}(t), P^{k}(t)$, and $P^{0}(t)(t \in J)$ denote the solutions of (12) and (13) corresponding to $\left\{\bar{x}_{0}^{k}, P_{0}^{k}, \tilde{A}^{k}, \tilde{\sigma}^{k}, Q^{k}, \bar{C}^{k}\right\}$ and $\left\{\bar{x}_{0}^{0}, P_{0}^{0}, \tilde{A}^{0}, \tilde{\sigma}^{0}, Q^{0}, \tilde{C}^{0}\right\}$. Then $\hat{x}^{k}(t) \rightarrow \hat{x}^{0}(t)$ and $P^{k}(t) \rightarrow P^{0}(t)$ uniformly in $t \in J$ as $k \rightarrow \infty$.

Proof. Consider

$$
\begin{array}{lll}
\frac{\mathrm{d}}{\mathrm{d} t} \hat{l}^{k}(t)=\bar{A}^{k}(t) \hat{x}^{k}(t)+\bar{C}^{k}(t) \beta(t) & (t \in J), & \hat{x}^{k}(0)=\bar{x}_{0}^{k} \\
\frac{\mathrm{d}}{\mathrm{d} t} \hat{l}^{0}(t)=\bar{A}^{0}(t) \hat{x}^{0}(t)+\tilde{C}^{0}(t) \beta(t) & (t \in J), & \hat{x}^{0}(0)=\bar{x}_{0}^{0}
\end{array}
$$

One can easily verify that

$$
\begin{aligned}
\left|\hat{x}^{k}(t)-\hat{x}^{0}(t)\right| \leqslant & \left|\bar{x}_{0}^{k}-\bar{x}_{0}^{0}\right|+\int_{0}^{t}\left\|\tilde{A}^{k}(\theta)\right\|\left|\hat{x}^{k}(\theta)-\hat{x}^{0}(\theta)\right| \mathrm{d} \theta \\
& +\int_{0}^{t}\left\|\tilde{A}^{k}(\theta)-\tilde{A}^{0}(\theta)\right\|\left|\hat{x}^{0}(\theta)\right| \mathrm{d} \theta+\int_{0}^{t}\left\|\tilde{C}^{k}(\theta)-\tilde{C}^{0}(\theta)\right\||\beta(\theta)| \mathrm{d} \theta .
\end{aligned}
$$

Using Assumptions (A1) and (A2) and noting that $\sup _{t e J}\left|\hat{x}^{0}(t)\right|=c_{1}<\infty$ ( $\hat{x}^{0}$ being a solution of the differential equation) and $\sup _{t e s}|\beta(t)|=c_{2}<\infty$, it follows from the above inequality and Gronwall's lemma that

$$
\begin{aligned}
\sup _{t \in f}\left|\hat{x}^{k}(t)-\hat{x}^{0}(t)\right| \leqslant\left(\left|\bar{x}_{0}^{k}-\bar{x}_{0}^{0}\right|\right. & +c_{1} \int_{0}^{t_{1}}\left\|\tilde{A}^{k}(\theta)-\tilde{A}^{0}(\theta)\right\| \mathrm{d} \theta \\
& \left.+c_{2} \int_{0}^{t_{1}}\left\|\tilde{C}^{k}(\theta)-\tilde{C}^{0}(\theta)\right\| \mathrm{d} \theta\right) \exp \left(\int_{0}^{t_{1}} K(\theta) \mathrm{d} \theta\right) .
\end{aligned}
$$

Since $K \in L_{1}^{\text {loc }}$, it follows by the hypotheses of the theorem and dominated convergence that the right-hand side of (42) converges to zero as $k \rightarrow \infty$. Hence we conclude that $\hat{x}^{k}(t) \rightarrow \hat{x}^{0}(t)$ uniformly in $t \in J$. It remains to show that $P^{k}(t) \rightarrow P^{0}(t)$ uniformly in $t \in J$. For this, consider

$$
\left.\begin{array}{rl}
\frac{\mathrm{d}}{\mathrm{d} t} P^{k}(t) & =\tilde{A}^{k}(t) P^{k}(t)+P^{k}(t)\left[\tilde{A}^{k}(t)\right]^{\top}+\tilde{\sigma}^{k}(t) Q^{k}(t)[\tilde{\sigma}(t)]^{\top}+\tilde{C}^{k}(t) \Gamma(t)\left[\tilde{C}^{k}(t)\right]^{\top}, \\
P^{k}(0) & =P_{0}^{k}, \\
\frac{\mathrm{d}}{\mathrm{d} t} P^{0}(t) & =\tilde{A}^{0}(t) P^{0}(t)+P^{0}(t)\left[\tilde{A}^{0}(t)\right]^{\top}+\tilde{\sigma}^{0}(t) Q^{0}(t)\left[\tilde{\sigma}^{0}(t)\right]^{\top}+\tilde{C}^{0}(t) \Gamma(t)\left[\tilde{C}^{0}(t)\right]^{\top}, \\
P^{0}(0) & =P_{0}^{0}
\end{array}\right\}
$$

for all $t \in J$. Then one can easily verify that

$$
\begin{aligned}
\left\|P^{k}(t)-P^{0}(t)\right\| \leqslant & \left\|P_{0}^{k}-P_{0}^{0}\right\|+2 \int_{0}^{t}\left\|\bar{A}^{k}(\theta)\right\|\left\|P^{k}(\theta)-P^{0}(\theta)\right\| \mathrm{d} \theta \\
& +2 \int_{0}^{t}\left\|P^{0}(\theta)\right\|\left\|\tilde{A}^{k}(\theta)-\bar{A}^{0}(\theta)\right\| \mathrm{d} \theta
\end{aligned}
$$




$$
\begin{aligned}
& +\int_{0}^{t}\left[\left\|\tilde{\sigma}^{k}(\theta)\right\|\left\|Q^{k}(\theta)\right\|+\left\|\tilde{\sigma}^{0}(\theta)\right\|\left\|Q^{0}(\theta)\right\|\right]\left\|\tilde{\sigma}^{k}(\theta)-\tilde{\sigma}^{0}(\theta)\right\| \mathrm{d} \theta \\
& +\int_{0}^{t}\left\|\tilde{\sigma}^{k}(\theta)\right\|\left\|\tilde{\sigma}^{0}(\theta)\right\|\left\|Q^{k}(\theta)-Q^{0}(\theta)\right\| \mathrm{d} \theta \\
& +\int_{0}^{t}\|\Gamma(\theta)\|\left[\left\|\tilde{C}^{k}(\theta)\right\|+\left\|\tilde{C}^{0}(\theta)\right\|\right]\left\|\tilde{C}^{k}(\theta)-\bar{C}^{0}(\theta)\right\| \mathrm{d} \theta
\end{aligned}
$$

for all $t \in J$. Using the Assumptions (A1) and (A2) and the fact that $\sup _{\text {reJ }}\left\|P^{0}(t)\right\|<\infty$, it follows from Gronwall's lemma that

$$
\begin{aligned}
\sup _{t e} & \left\|P^{k}(t)-P^{0}(t)\right\| \\
\leqslant & \left(\left\|P_{0}^{k}-P_{0}^{0}\right\|+2 c_{3} \int_{0}^{t_{1}}\left\|\tilde{A}^{k}(\theta)-\tilde{A}^{0}(\theta)\right\| \mathrm{d} \theta\right. \\
& +c_{4} \int_{0}^{t_{1}}\left\|\tilde{\sigma}^{k}(\theta)-\tilde{\sigma}^{0}(\theta)\right\| \mathrm{d} \theta+c_{5} \int_{0}^{t_{1}}\left\|Q^{k}(\theta)-Q^{0}(\theta)\right\| \mathrm{d} \theta \\
& \left.+c_{6} \int_{0}^{t_{1}}\left\|\tilde{C}^{k}(\theta)-\tilde{C}^{0}(\theta)\right\| \mathrm{d} \theta\right) \exp \left(2 \int_{0}^{t_{1}} K(\theta) \mathrm{d} \theta\right),
\end{aligned}
$$

where $c_{3}, c_{4}, c_{5}$, and $c_{6}$ are positive constants. Using arguments similar to those given above, it follows from the dominated convergence theorem that the right-hand side of (46) converges to zero in the limit. This completes the proof.

In the following section, we utilize the results of Theorem 3.1 to develop a recursive scheme on the basis of which the estimate $\hat{x}\left(t_{k} \mid t_{k}\right)$ and corresponding covariance $P\left(t_{k} \mid t_{k}\right)$ can be determined for all $t_{k}$. Then, based on the navigation system model proposed in [10], we present a numerical example to illustrate the effectiveness of the proposed filter.

\section{Algorithm and numerical simulations}

In this section, we present, on the basis of Theorem 3.1, an iterative scheme for computing the estimate $\hat{x}\left(t_{k} \mid t_{k}\right)$ and the corresponding covariance $P\left(t_{k} \mid t_{k}\right)$ for all $t_{k}$. This algorithm is then applied to a navigation system model to illustrate the results of Theorem 3.1 .

Consider the differential equation (12) and let $\varphi(t, \tau)(\tau \leqslant t)$ denote the corresponding transition operator. Then, for any $t \in\left[t_{k-1}, t_{k}\right]$, the solution of (12) is given by

$$
\hat{x}(t \mid t)=\hat{x}\left(t \mid t_{k-1}\right)=\varphi\left(t, t_{k-1}\right) \hat{x}\left(t_{k-1} \mid t_{k-1}\right)+\int_{0}^{t} \varphi(t, \theta) \tilde{C}(\theta) \beta(\theta) \mathrm{d} \theta,
$$

where $\beta(t)=\beta^{y}(t)(t \geqslant 0)$ is given by (14). Assuming that $\Delta_{k} \equiv\left(t_{k}-t_{k-1}\right)$ is 
sufficiently small, it follows from the above equation that

$$
\hat{f}\left(t_{k} \mid t_{k-1}\right) \cong \varphi\left(t_{k}, t_{k-1}\right) \mathcal{f}\left(t_{k-1} \mid t_{k-1}\right)+\varphi\left(t_{k}, t_{k-1}\right) \tilde{C}\left(t_{k-1}\right) \beta\left(t_{k-1}\right) \Delta_{k} .
$$

Similarly, one can easily verify that the solution of (13) can be written as

$$
\begin{aligned}
P\left(t_{k} \mid t_{k-1}\right) \simeq & \varphi\left(t_{k}, t_{k-1}\right) P\left(t_{k-1} \mid t_{k-1}\right) \varphi^{\top}\left(t_{k} \mid t_{k-1}\right) \\
& +\varphi\left(t_{k} \mid t_{k-1}\right) \tilde{\sigma}\left(t_{k-1}\right) \tilde{Q}\left(t_{k-1}\right) \tilde{\sigma}^{\top}\left(t_{k-1}\right) \varphi^{\top}\left(t_{k}, t_{k-1}\right) \\
& +\varphi\left(t_{k}, t_{k-1}\right) \tilde{C}\left(t_{k-1}\right) \tilde{\Gamma}\left(t_{k-1}\right) \tilde{C}^{\top}\left(t_{k-1}\right) \varphi^{\top}\left(t_{k}, t_{k-1}\right),
\end{aligned}
$$

where $\widetilde{Q}\left(t_{k-1}\right)=\Delta_{k} Q\left(t_{k-1}\right)$ and $\tilde{\Gamma}\left(t_{k-1}\right)=\Delta_{k} \Gamma\left(t_{k-1}\right)$.

With the help of the above set of (discrete) equations, we now present the following algorithm for computing $\mathcal{L}\left(t_{k} \mid t_{k}\right)$ and $P\left(t_{k} \mid t_{k}\right)$ for all $t_{k}$.

\subsection{Algorithm}

1. Set $k=1$.

2. Given $x\left(t_{k}\right), y\left(t_{k}\right),\left\{\lambda_{i, j}: i, j=1,2, \ldots, M\right\}, \bar{A}\left(t_{k}\right), \tilde{\sigma}\left(t_{k}\right), \bar{C}\left(t_{k}\right), Q\left(t_{k}\right), H\left(t_{k}\right)$, and $R\left(t_{k}\right)$, obtain $x\left(t_{k+1}\right)$ and $y\left(t_{k+1}\right)$.

3. Compute the conditional probabilities $p_{l}\left(t_{k+1}\right)=p_{l}^{y}\left(t_{k+1}\right)$ using the following logic:

(i) If $k=1$, set $p_{i}\left(t_{1}\right)=p_{i}(0)(1 \leqslant i \leqslant M)$, where $p_{i}(0)$ denotes the initial distribution.

(ii) If $k>1$, compute $\left|y\left(t_{k+1}\right)-y\left(t_{k}\right)\right|$ and

(a) if $\left|y\left(t_{k+1}\right)-y\left(t_{k}\right)\right| \geqslant c$, where $c$ is a given threshold, obtain new values for $p_{i}\left(t_{k+1}\right) \quad(1 \leqslant i \leqslant M)$;

(b) if $\left|y\left(t_{k+1}\right)-y\left(t_{k}\right)\right|<c$, set $p_{i}\left(t_{k+1}\right)=p_{i}\left(t_{k}\right)(1 \leqslant i \leqslant M)$.

4. Using (14) and (47), compute $\beta\left(t_{k+1}\right) \boxminus \beta^{y}\left(t_{k+1}\right)$ and $f\left(t_{k+1} \mid t_{k}\right)$.

5. Given $S\left(t_{k}\right)$, compute $S\left(t_{k+1}\right)$ using the relation

$$
S\left(t_{k+1}\right) \simeq S\left(t_{k}\right)+\left(t_{k+1}-t_{k}\right) \Lambda S\left(t_{k}\right),
$$

where the elements of $\Lambda$ are $\left\{\lambda_{l, j}: i, j=1,2, \ldots, M\right\}$.

6. Given $\theta\left(t_{k}\right)$, compute $\theta\left(t_{k+1}\right)$ using the relation

$$
\theta\left(t_{k+1}\right)=S\left(t_{k+1}\right) \theta\left(t_{k}\right)
$$

7. Obtain $\Gamma\left(t_{k+1}\right)$ using (15).

8. Given $P\left(t_{k} \mid t_{k}\right)$, use (48) to obtain $P\left(t_{k+1} \mid t_{k}\right)$.

9. Compute the filter gain $G^{0}\left(t_{k+1}\right)$ using (20).

10. Using (18) and (19), compute $\hat{x}\left(t_{k+1} \mid t_{k+1}\right)$ and $P\left(t_{k+1} \mid t_{k+1}\right)$.

11. If $k<N$, set $k=k+1$ and go to step 2; otherwise stop.

\subsection{Navigation Example}

In this section, we apply the algorithm presented above to a navigation system in which Loran-C measurements are used to estimate the errors in a dead reckoning (DR) system. We first present the standard model for a DR system (see e.g. [3]) along with the Loran-C error model that has been proposed in [10] and the corresponding observation model. Then, with the help of Theorem 3.1 we obtain the corresponding filter equations on the basis of which the numerical results shown in Figs. 2-7 were obtained. 
DR Error Model The DR error model is given by $[3,9,10]$

$$
\left.\begin{array}{l}
\frac{\mathrm{d}}{\mathrm{d} t} \tilde{\lambda}(t)=V_{\mathrm{N}}(t), \\
\frac{\mathrm{d}}{\mathrm{d} t} \tilde{L}(t)=V_{\mathrm{E}}(t), \\
\mathrm{d} V_{\mathrm{N}}(t)=-\frac{1}{T_{\mathrm{c}}} V_{\mathrm{N}}(t) \mathrm{d} t+\mathrm{d} W_{1}(t), \\
\mathrm{d} V_{\mathrm{E}}(t)=-\frac{1}{T_{c}} V_{\mathrm{E}}(t) \mathrm{d} t+\mathrm{d} W_{2}(t),
\end{array}\right\}
$$

where $T_{c}(>0)$ denotes the ocean current correlation time, $(\bar{\lambda}, \bar{L})$ are the latitude and longitude errors, and $\left(V_{N}, V_{E}\right)$ are the northern and eastern components of the velocity error. The processes $W_{1}$ and $W_{2}$ are two (one-dimensional) independent Wiener processes.

Loran-C Error Model Under the assumption that the Loran-C receiver uses a master and two slave transmitters, the time difference error is modelled as

$$
\xi_{i}(t)=\xi_{i}^{\prime}(t)+\xi_{i}^{\prime \prime}(t) \quad(t \geqslant 0 ; i=1,2),
$$

where $\xi_{i}^{\prime}(i=1,2)$ is governed by the following (linear) stochastic differential equations $[3,9,10]$ :

$$
\left.\begin{array}{l}
\mathrm{d} \xi_{1}^{\prime}(t)=-\frac{1}{T_{\mathrm{L}}} \xi_{1}^{\prime}(t) \mathrm{d} t+\mathrm{d} W_{3}(t) \\
\mathrm{d} \xi_{2}^{\prime}(t)=-\frac{1}{T_{\mathrm{L}}} \xi_{2}^{\prime}(t) \mathrm{d} t+\mathrm{d} W_{4}(t)
\end{array}\right\} \quad(t \geqslant 0),
$$

where $T_{L}>0$ and $\left(W_{3}, W_{4}\right)$ are two independent Wiener processes. The process $\xi_{i}^{\prime \prime}(i=1,2)$ is a pure jump process (representing the cycle selection error) governed by [10]

$$
\begin{aligned}
\mathrm{d} \xi_{i}^{\prime \prime}(t)= & \gamma\left\{1\left(\xi_{i}^{\prime \prime}\left(t^{-}\right)=-\gamma l\right) N_{1,2}^{\prime}(\mathrm{d} t)-1\left(\xi_{i}^{\prime \prime}\left(t^{-}\right)=\gamma l\right) N_{2 l+1,2 \lambda}^{i}(\mathrm{~d} t)\right. \\
& \left.+\sum_{j=2}^{2 l} 1\left(\xi_{i}^{\prime \prime}\left(t^{-}\right)=(j-l-1) \gamma\right)\left[N_{j, j+1}^{t}(\mathrm{~d} t)-N_{j, j-1}^{l}(\mathrm{~d} t)\right]\right\}
\end{aligned}
$$

for all $t \geqslant 0$ and $i=1,2$, where $\gamma>0$ and $\left\{N_{1,2}^{i}, N_{2,1}^{i}, N_{2,3}^{i}, \ldots, N_{2 t+1,2 u}^{i}\right\}$ are independent Poisson processes with mean $\left\{\lambda_{1,2}^{i}, \lambda_{2,1}^{i}, \lambda_{2,3}^{i}, \ldots, \lambda_{2 l+1,21}^{i}\right\}(i=1,2)$. The state transition diagram for the process $\xi_{i}^{\prime \prime}(i=1,2)$ is shown in Fig. 1, with $e_{i}=\gamma i(-l \leqslant i \leqslant l)$. Defining

$x=\left[\bar{\lambda}, \tilde{L}, V_{N}, V_{E}, \xi_{1}^{\prime}, \xi_{1}^{n}, \xi_{2}^{\prime}, \xi_{2}^{n}\right]^{\top}, \quad W=\left[W_{1}, W_{2}, W_{3}, W_{4}\right]^{\top}, \quad N=\left[N^{1}, N^{2}\right]^{\top}$, with

$$
\begin{aligned}
N^{i}(\mathrm{~d} t)= & 1\left(\xi_{i}^{\prime \prime}\left(t^{-}\right)=-\gamma l\right) N_{1,2}^{i}(\mathrm{~d} t)-1\left(\xi_{i}^{\prime \prime}\left(t^{-}\right)=\gamma l\right) N_{2 t+1,2}^{i}(\mathrm{~d} t) \\
& +\sum_{j=2}^{2 l} 1\left(\xi_{i}^{\prime \prime}\left(t^{-}\right)=(j-l-1) \gamma\right)\left[N_{j, j+1}^{\prime}(\mathrm{d} t)-N_{j, j-1}^{i}(\mathrm{~d} t)\right] \quad(i=1,2),
\end{aligned}
$$


we can write the overall navigation system (44)-(51) as

$$
\mathrm{d} x(t)=\tilde{A} x(t) \mathrm{d} t+\tilde{\sigma} \mathrm{d} W(t)+\tilde{C} N(\mathrm{~d} t) .
$$

Here the (time-invariant) matrices $\tilde{A}, \tilde{\sigma}$, and $\tilde{C}$ are given by

$$
\begin{gathered}
\tilde{A}=\left[\begin{array}{cccccccc}
0 & 0 & 1 & 0 & 0 & 0 & 0 & 0 \\
0 & 0 & 0 & 1 & 0 & 0 & 0 & 0 \\
0 & 0 & -1 / T_{\mathrm{c}} & 0 & 0 & 0 & 0 & 0 \\
0 & 0 & 0 & -1 / T_{\mathrm{c}} & 0 & 0 & 0 & 0 \\
0 & 0 & 0 & 0 & -1 / T_{\mathrm{L}} & 0 & 0 & 0 \\
0 & 0 & 0 & 0 & 0 & 0 & 0 & 0 \\
0 & 0 & 0 & 0 & 0 & 0 & -1 / T_{\mathrm{L}} & 0 \\
0 & 0 & 0 & 0 & 0 & 0 & 0 & 0
\end{array}\right] \\
\tilde{\sigma}=\left[\begin{array}{cccc}
0 & 0 & 0 & 0 \\
0 & 0 & 0 & 0 \\
1 & 0 & 0 & 0 \\
0 & 1 & 0 & 0 \\
0 & 0 & 1 & 0 \\
0 & 0 & 0 & 0 \\
0 & 0 & 0 & 1 \\
0 & 0 & 0 & 0
\end{array}\right], \quad \tilde{C}=\left[\begin{array}{cc}
0 & 0 \\
0 & 0 \\
0 & 0 \\
0 & 0 \\
0 & 0 \\
\gamma & 0 \\
0 & 0 \\
0 & \gamma
\end{array}\right]
\end{gathered}
$$

Observation Model Under the assumption that the Loran-C receiver uses a master and two slave transmitters, the observation model is given by [3]

$$
y\left(t_{k}\right)=H x\left(t_{k}\right)+\left[V\left(t_{k}\right)-V\left(t_{k-1}\right)\right]
$$

where $V$ is a Wiener process independent of $W$ and $N$, representing the receiver noise and the matrix $H$ is given by

$$
H=\left[\begin{array}{lllllllll}
\left(\cos \psi_{M}-\cos \psi_{\mathrm{S}_{1}}\right) & \left(\sin \psi_{\mathrm{M}}-\sin \psi_{\mathrm{S}_{1}}\right) & 0 & 0 & 1 & 1 & 0 & 0 \\
\left(\cos \psi_{\mathrm{M}}-\cos \psi_{\mathrm{S}_{2}}\right) & \left(\sin \psi_{\mathrm{M}}-\sin \psi_{\mathrm{S}_{2}}\right) & 0 & 0 & 0 & 0 & 1 & 1
\end{array}\right]
$$

Here $\psi_{M}$ denotes the bearing of the ship to the Loran-C master transmitter and $\psi_{\mathrm{S}}(i=1,2)$ is the bearing to the slave transmitter.

Filter Equations Setting $M=2 l+1$ and $e_{j}=j(-l \leqslant j \leqslant l)$ and noting that $\beta=\left[\beta_{1}, \beta_{2}\right]^{\top}$ and $\Gamma$ is a diagonal matrix, it follows from (14) and (15) that

$$
\begin{aligned}
& \beta_{i}(t)=\sum_{j=2}^{M-1}\left(\lambda_{j, j+1}^{l}-\lambda_{j, j-1}^{i}\right) p_{j}^{i}(t)+\lambda_{1,2}^{i} p_{1}^{i}(t)-\lambda_{M, M-1}^{i} p_{M}^{l}(t), \\
& \Gamma_{i l}(t)=\sum_{j=2}^{M-1}\left(\lambda_{j, j+1}^{i}+\lambda_{j, j-1}^{l}\right) \Theta_{j}^{i}(t)+\lambda_{1,2}^{i} \Theta_{1}^{i}(t)+\lambda_{M, M-1}^{i} \Theta_{M}(t),
\end{aligned}
$$



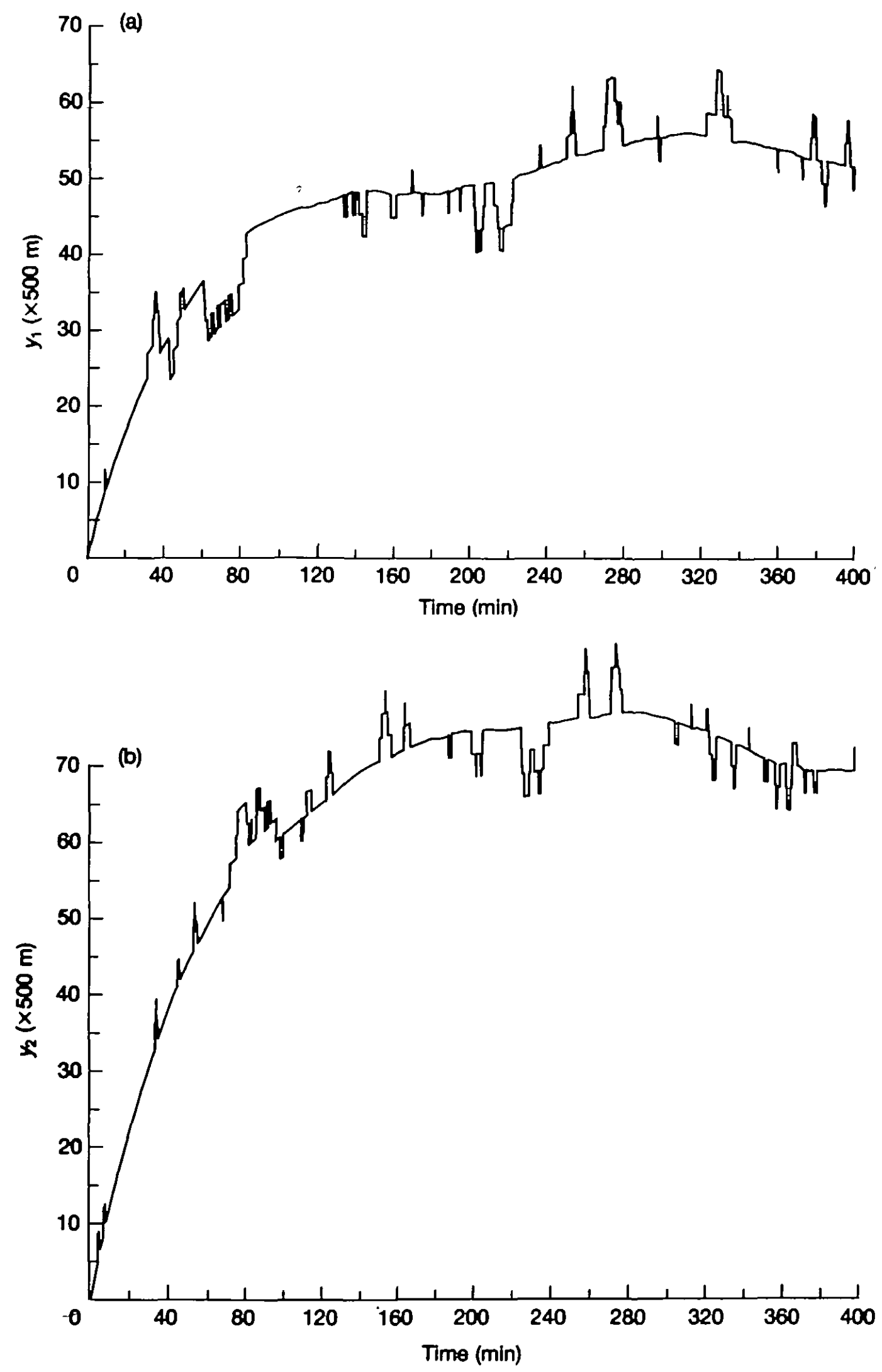

Fio. 2. (a) Observation process $y_{1}$; (b) observation process $y_{2}$. 


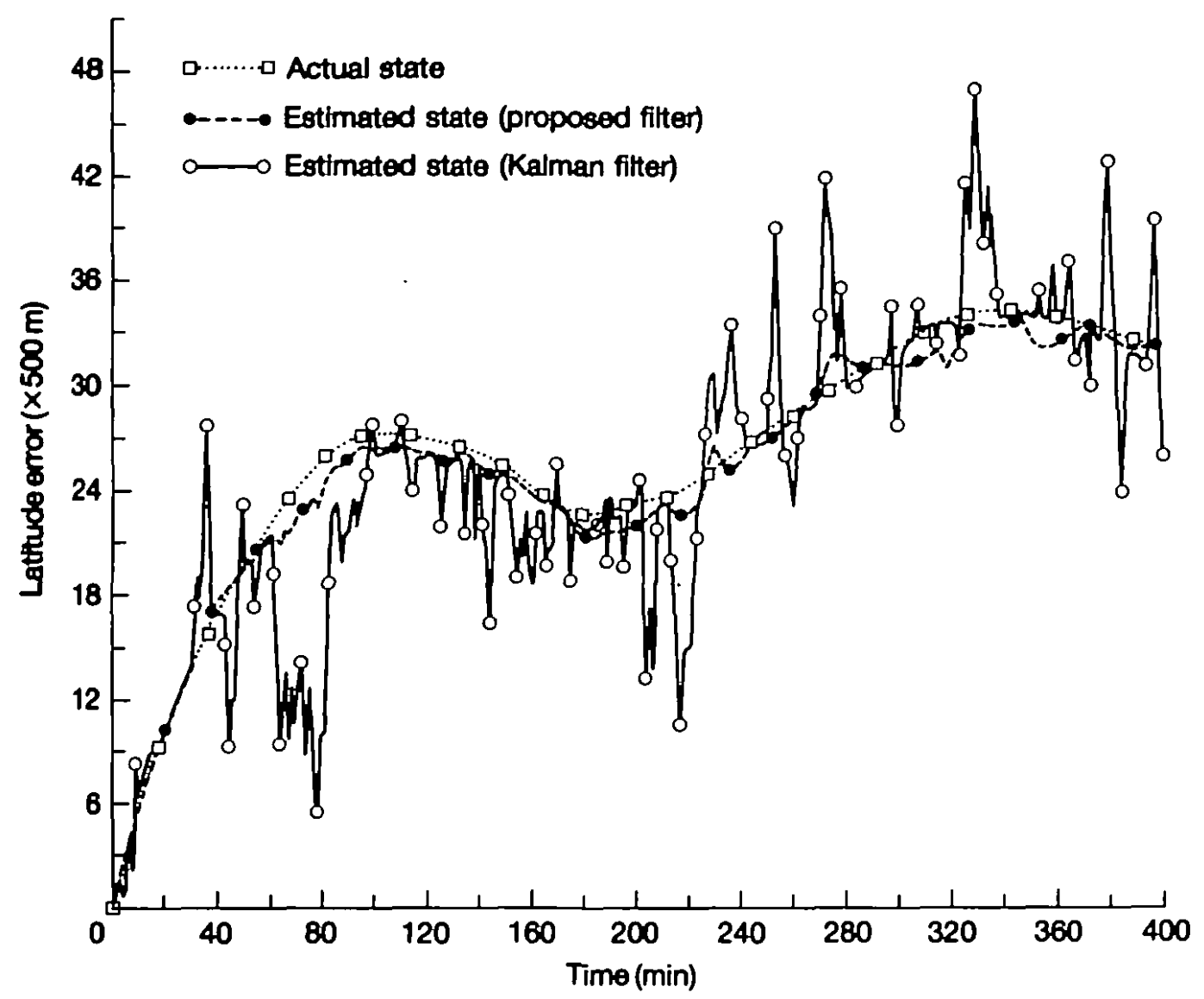

FIo. 3. Latitude error.

for $i=1,2$. Now, using equations (54) and (55) and the navigation system parameters $\bar{A}, \tilde{\sigma}$, and $\tilde{C}$, one can easily obtain the corresponding filter equations using Theorem 3.1.

Numerical Simulations Using the above algorithm and equations (18)-(20), (47), (48), (54), and (55), we have generated the processes $x\left(t_{k}\right)$ and $y\left(t_{k}\right)$ and computed the corresponding estimates using the proposed filter as well as the Kalman filter. The numerical results presented in Figs. 2-7 are for the case where $M=7, \gamma=3000 \mathrm{~m}$, and

$$
\begin{aligned}
& \lambda_{1,2}^{l}=0.8, \quad \lambda_{2,3}^{l}=0.65, \lambda_{3,4}^{i}=0.6, \quad \lambda_{4,5}^{l}=0.055, \lambda_{5,6}^{i}=0.44, \lambda_{8,7}^{i}=0.45, \\
& \lambda_{2,1}^{l}=0.43, \lambda_{3,2}^{l}=0.43, \lambda_{4,3}^{i}=0.05, \lambda_{5,4}^{l}=0.6, \quad \lambda_{6,5}^{i}=0.7, \quad \lambda_{7,6}^{l}=0.85,
\end{aligned}
$$

for $i=1,2$. From these results, it is clear that, whenever the proposed filter is used, the behaviour of the estimated state is very similar to that of the actual state and their values are fairly close (see Figs. 2-7). It is also clear from Fig. 7 that, whenever the Loran-C errors jump from one state to another, the proposed filter tends to follow these jumps with reasonable accuracy. On the other hand, the Kalman filter does not respond at all to these jumps. Indeed, from these results, it is clear that the response of the proposed filter to the rapid changes of the 


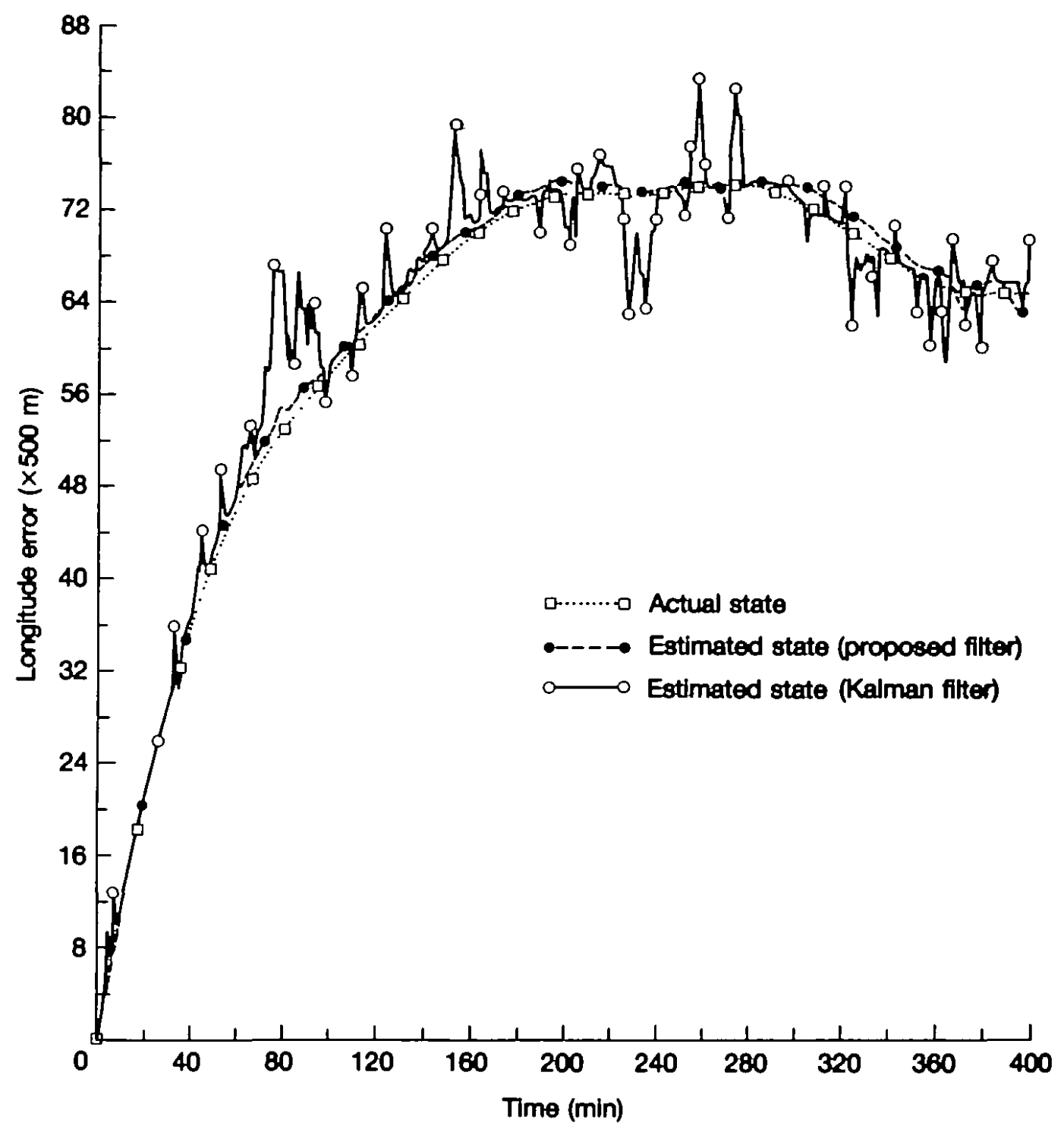

Fig. 4. Latitude error.

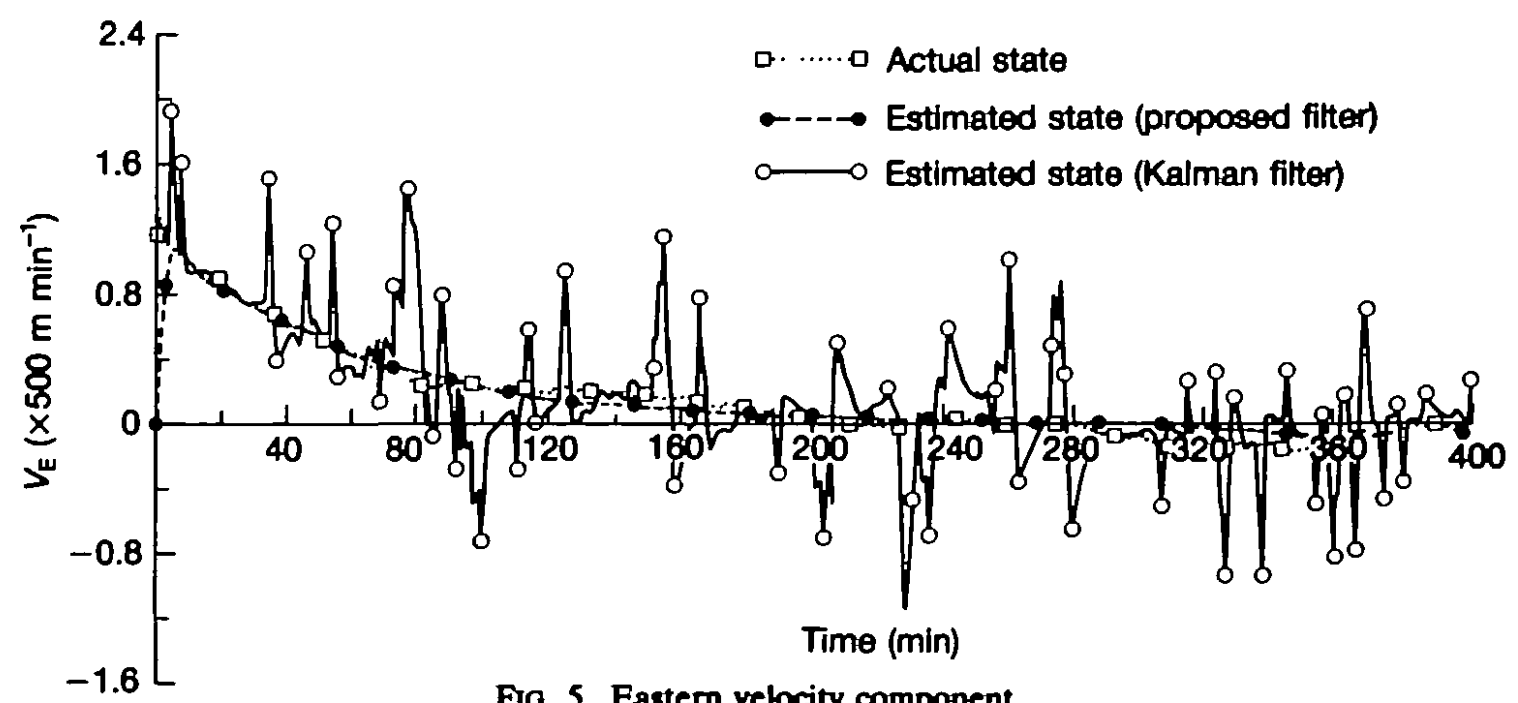

Fio. 5. Eastern velocity component. 


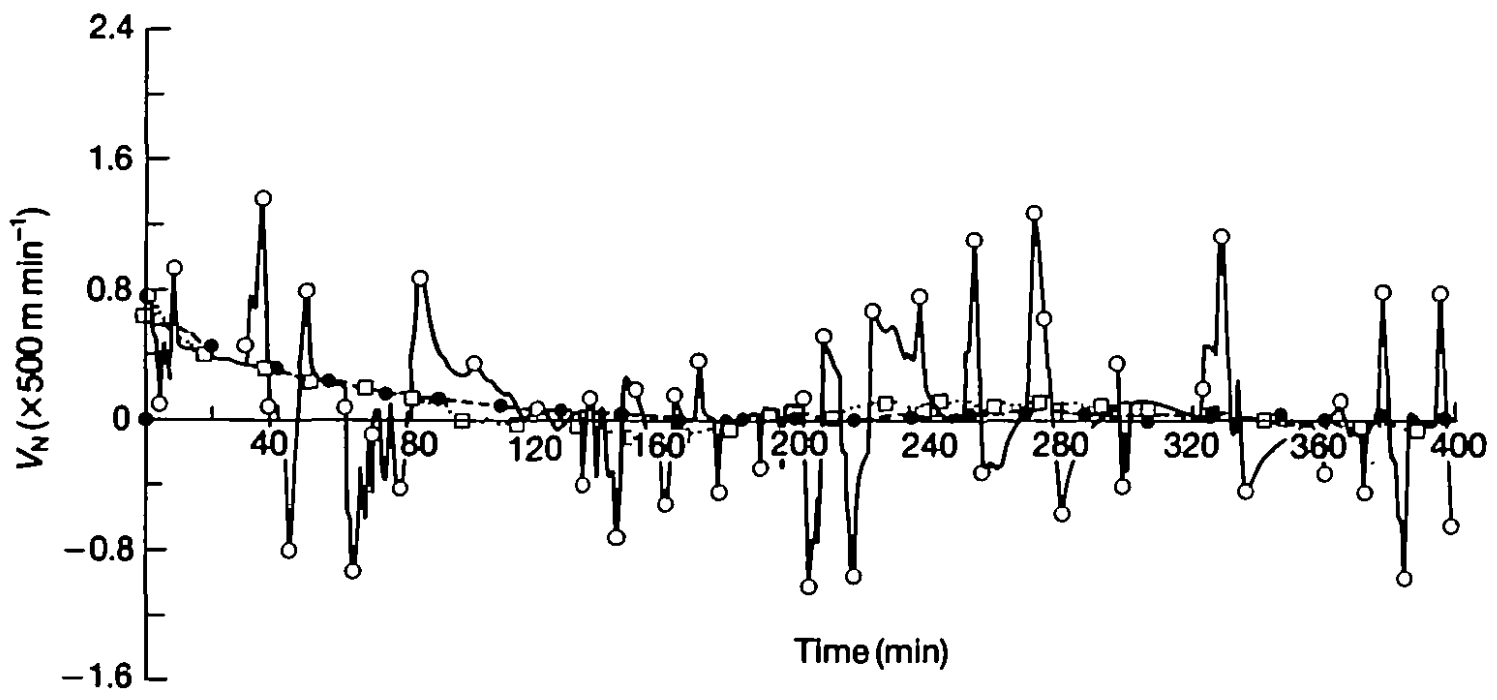

Fro. 6. Northern velocity component.
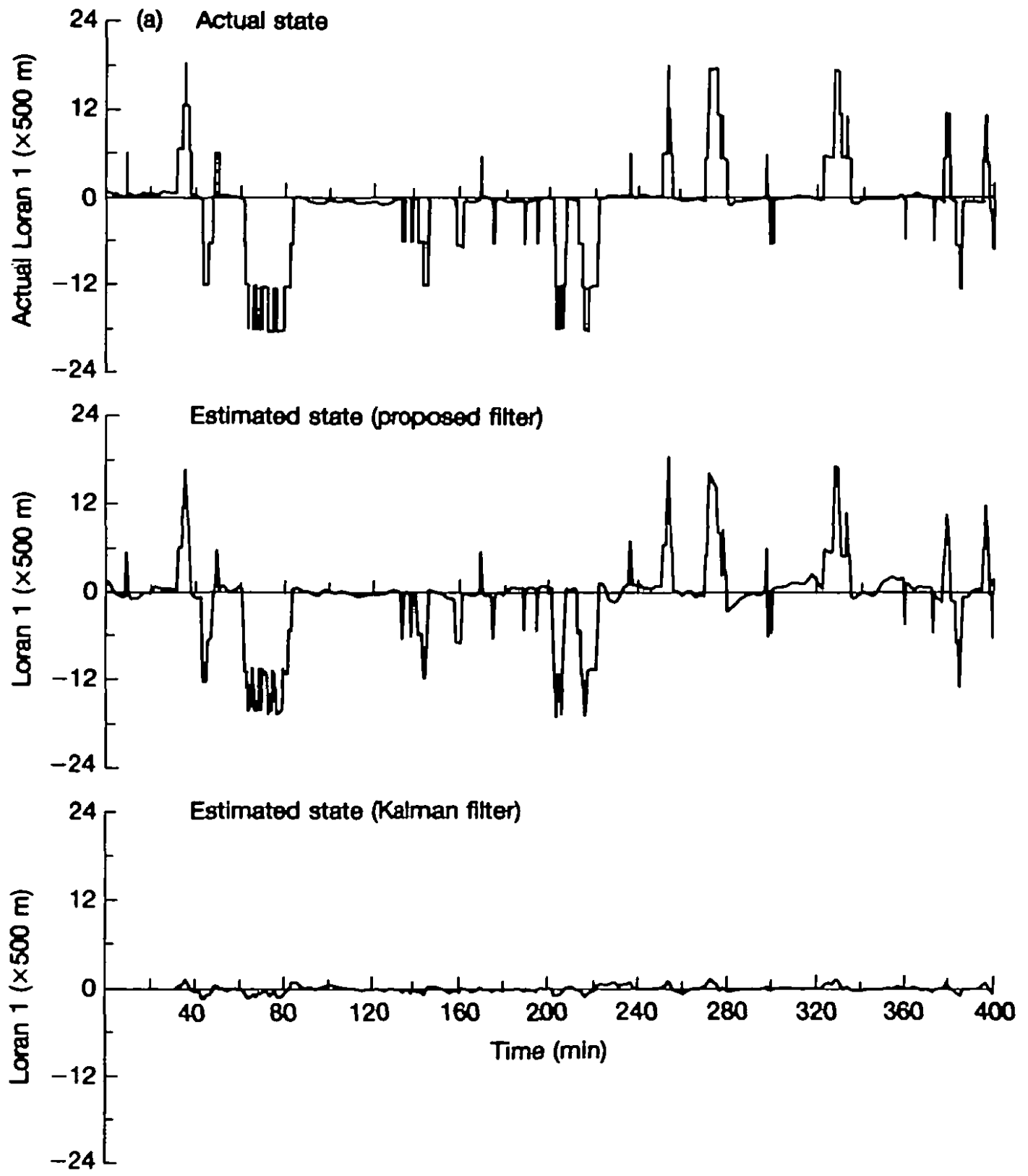

Fio. 7. (a) Loran-C error $\xi_{1}$; (b) Loran-C error $\xi_{2}$. 


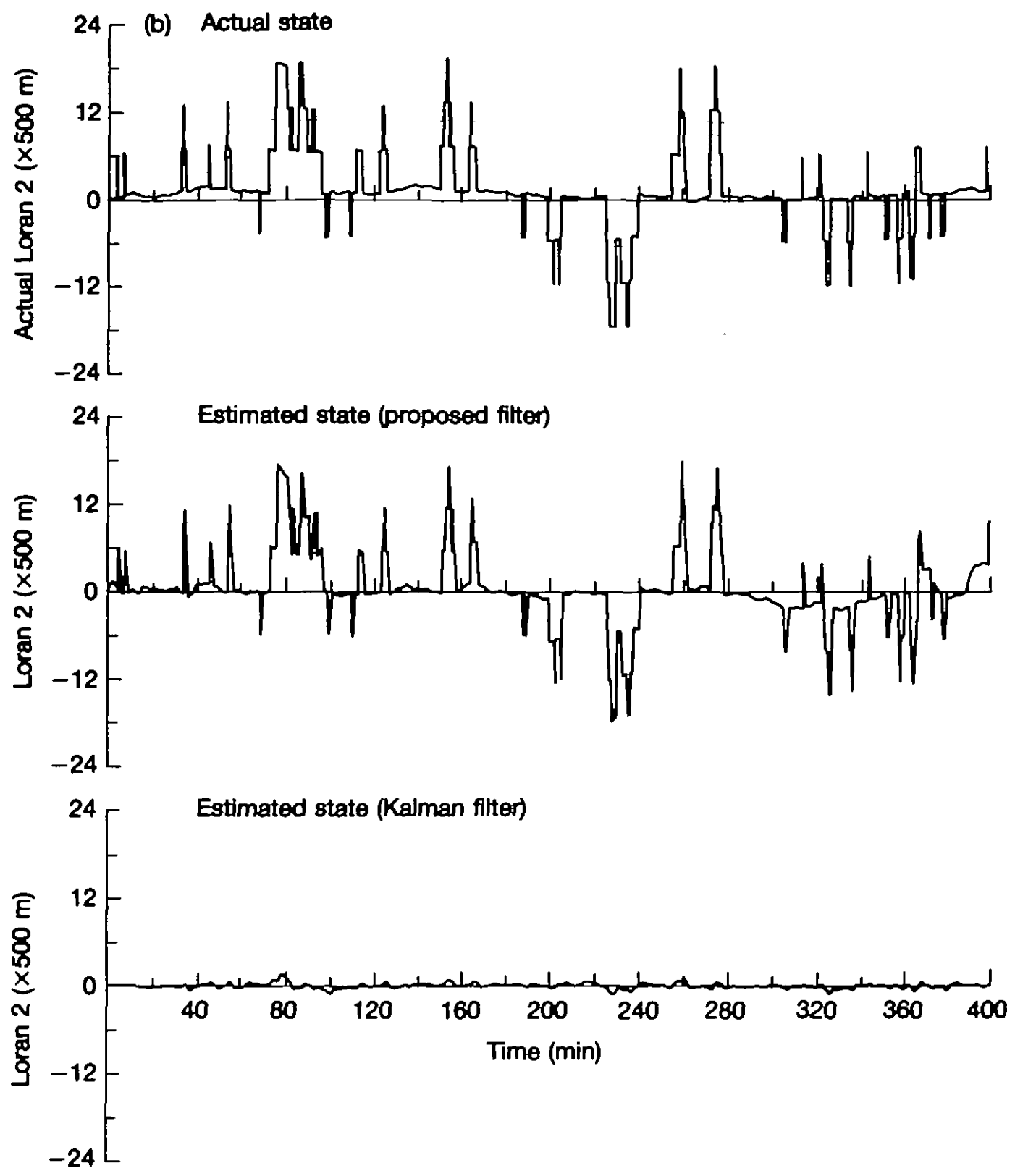

FIG. 7 (contd).

Loran-C error is much better than that of the Kalman filter, and hence one expects better estimates of position and velocity. Finally, it should be noted that similar results have been obtained for the case where an Omega receiver (which is modelled in a similar way to Loran-C) is used along with DR and Loran-C.

\section{Conctusions}

In this paper, we have considered the filtering problem for a class of jump processes with discrete observations. Under the assumption that the elements of the covariance matrices of state and observation noise are very small compared 
with the size of jump of the state process, we have obtained a recursive linear unbiased minimum variance filter. Using these filter equations, we have also studied the question of continuous dependence of the filter on system parameters. Finally, we have presented a numerical example, which is based on a navigation system, to illustrate the effectiveness of the proposed filter. From this example, we have seen that the estimated states computed via the proposed filter are closer to the actual states than those obtained by use of the Kalman filter. Further, we have also observed that the proposed filter is able to follow the rapid changes of the Loran- $\mathrm{C}$ error more closely than the Kalman filter, and hence better estimates for ship (or aircraft) position and velocity can be obtained.

\section{REFERENCES}

1. Aнmеd, N. U. 1988 Elements of Finite-Dimensional Systems and Control Theory. Harlow: Longman.

2. AHMED, N. U. 1988 Optimization and Identification of Systems Governed by Evolution Equations on Banach Space, Pitman Research Notes in Mathematical Sciences 184. Harlow: Longman.

3. Dabbous, T. E., Ahmed, N. U., McMmlan, J. C., \& Liano, D. F. 1988 Filtering of discontinuous processes arising in marine integrated navigation systems. IEEE Trans. Aerospace Electron. Syst. 24, 85-102.

4. Ahmed, N. U., \& Dabmous, T. E. 1983 Modelling and on-line control and reliability dynamics of large scale interconnected power systems. Int. J. Syst. Sci. 14, 1321-53.

5. Ahmed, N. U., \& DABbous, T. E. 1986 Nonlinear filtering of systems governed by Ito differential equations with jump parameters. J. Math. Anal. Appl. 115, 76-92.

6. Dabeous, T. E., \& Ahmed, N. U. 1981 Nonlinear filtering of diffusion processes with discontinuous observations. J. Stochast. Anal. Appl. 2(4), 87-106.

7. Kushner, H. J. 1967 Dynamical equations for optimal nonlinear filtering. J. Diff. Eqn. 3, 179-190.

8. ZAKAI, M. 1969 On the optimal filtering of diffusion processes. Z. Wahrsch. Verw. Geb. 11, 230-43.

9. Ahmed, N. U., \& DABbous, T. E. 1985 Study of discontinuous processes in Omega and Loran-C radio signals. Report submitted to Defence Research and Establishment Ottawa, Ottawa, Canada.

10. Ahmed, N. U., \& Lim, S. S. 1987 Continuation of the study of discontinuous processes in Omega and Loran-C radio signals. Report submitted to Defence Research and Establishment Ottawa, Ottawa, Canada.

11. Jazwinski, A. H. 1970 Stochastic Processes and Filtering Theory. London: Academic Press.

12. SKorokhod, A. V. 1965 Studies in The Theory of Random Processes. Reading, MA: Addison-Wesley.

13. Boel, R., Varaiya, P., \& Wong, E. 1975 Martingales on jump processes II. SIAM J. Control Optim. 13, 1022-60.

14. Takeuchi, Y., \& AKASH, H. 1981 Nonlinear filtering formulas for discrete time observations. SIAM J. Control Optim. 19, 244-61. 